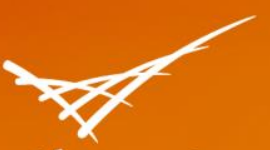

Pacific Northwest

NATIONAL LABORATORY

\title{
Characteristics and Performance of Existing Load Disaggregation Technologies
}

\section{April 2015}

ET Mayhorn

RS Butner MC Baechler
GP Sullivan

$\mathrm{H} \mathrm{HaO}$ 


\title{
DISCLAIMER
}

This report was prepared as an account of work sponsored by an agency of the United States Government. Neither the United States Government nor any agency thereof, nor Battelle Memorial Institute, nor any of their employees, makes any warranty, express or implied, or assumes any legal liability or responsibility for the accuracy, completeness, or usefulness of any information, apparatus, product, or process disclosed, or represents that its use would not infringe privately owned rights. Reference herein to any specific commercial product, process, or service by trade name, trademark, manufacturer, or otherwise does not necessarily constitute or imply its endorsement, recommendation, or favoring by the United States Government or any agency thereof, or Battelle Memorial Institute. The views and opinions of authors expressed herein do not necessarily state or reflect those of the United States Government or any agency thereof.

\author{
PACIFIC NORTHWEST NATIONAL LABORATORY \\ operated by \\ BATTELLE \\ for the \\ UNITED STATES DEPARTMENT OF ENERGY \\ under Contract DE-AC05-76RL01830
}

Printed in the United States of America
Available to DOE and DOE contractors from the Office of Scientific and Technical Information,
P.O. Box 62, Oak Ridge, TN 37831-0062;
ph: (865) 576-8401
fax: $(865) 576-5728$
email: reports@adonis.osti.gov

Available to the public from the National Technical Information Service

5301 Shawnee Rd., Alexandria, VA 22312

ph: (800) 553-NTIS (6847)

email: orders@ntis.gov <http://www.ntis.gov/about/form.aspx>

Online ordering: http://www.ntis.gov

This document was printed on recycled paper.

$(8 / 2010)$ 


\title{
Characteristics and Performance of Existing Load Disaggregation Technologies
}

\author{
ET Mayhorn \\ GP Sullivan \\ RS Butner \\ $\mathrm{H} \mathrm{HaO}$ \\ MC Baechler
}

April 2015

Prepared for

the U.S. Department of Energy

under Contract DE-AC05-76RL01830

Pacific Northwest National Laboratory

Richland, Washington 99352 



\section{Summary}

Non-intrusive load monitoring (NILM) or non-intrusive appliance load monitoring (NIALM) is an analytic approach used to disaggregate building loads based on a single metering point. This advanced load monitoring and disaggregation technique has the potential to provide an alternative solution to highpriced traditional sub-metering and enable innovative approaches for energy conservation, energy efficiency, and demand response. However, since the inception of the concept in the 1980s, evaluations of these technologies have focused on reporting performance accuracy without investigating sources of inaccuracies or fully understanding and articulating the meaning of the metrics used to quantify performance. As a result, the market for, as well as advances in, these technologies have been maturing slowly. So far, 18 active companies operating in the United States, United Kingdom, Ireland, and Canada have been identified to have commercially available or advanced prototypes of NILM devices.

To improve the market for these NILM technologies there has to be confidence that the deployment will lead to benefits. In reality, every end-user and application that this technology may enable does not require the highest levels of performance accuracy to produce benefits. Also, other important characteristics need to be considered, which may affect the appeal of NILM products to certain market targets (i.e., residential and commercial building consumers) and their suitability for particular applications. These characteristics include the following: 1) ease of use, that is, the level of expertise/bandwidth required to properly use the product; 2) ease of installation, that is, the level of expertise required to install the products along with hardware needs that affect product cost; and 3) ability to inform decisions and actions, that is, whether the energy outputs received by end-users (e.g., third-party applications, residential users, building operators, etc.) empower decisions and actions to be taken at time frames required for certain applications. Therefore, stakeholders, researchers, and other interested parties should be kept abreast of the evolving capabilities, uses, and characteristics of NILM technologies that make them attractive for certain building environments and different classes of end-users.

This report describes the performance of a few existing technologies that were evaluated as part of the Residential Building Stock Assessment (RBSA) owner-occupied test bed operated by the Northwest Energy Efficiency Alliance (NEEA) to understand the performance accuracy of current NILM products under realistic conditions. Based on this field study experience, the characteristics exhibited by the NILM products included in the assessment are also discussed in this report in terms of their ease of use, ease of installation, and ability to inform decisions and actions. The results of the analysis performed to investigate the accuracy of the participating NILM products in estimating energy use of individual appliances are also presented.

Evaluating the performance of disaggregation technologies is not trivial because the performance can be misunderstood and misinterpreted if objective metrics and protocols are not used. This became very apparent when examining real-world consumer data versus laboratory-generated or formulated data sets. Many of the loads being disaggregated by these technologies tend to be diverse and have unique characteristics. Moreover, vendors tend to have different methods of labeling, identifying, and reporting energy use for individual loads. After evaluating several candidate metrics, the "percent standard deviation explained" was finally selected to represent the accuracy of NILM energy estimates because it is able to quantify how well a NILM device is able to track the energy profiles of the individual loads. Performance evaluations were then divided into several stages to investigate the sources of inaccuracies caused by the labeling processes employed by each NILM device and the method used to estimate energy 
use. For example, through the process followed, it became evident that if the labeling and identification were improved in one of the technologies evaluated, the NILM device would do reasonably well ( $>70 \%$ on average) in estimating the energy use of major appliances that are considered resistive loads and have simple ON/OFF states. In addition, there are challenges related to recognizing multi-state loads (e.g., freezers and refrigerators) as a single load, resulting in lowered performance accuracy in estimating the energy use of these appliances ( $<30 \%$ on average).

The NEEA field study of existing NILM products has helped to highlight challenges associated with characterizing the performance of NILM technologies, the sources of inaccuracy, as well as development opportunities that should be explored help to enable useful applications. However, to eliminate the need for performing expensive large-scale field demonstrations to evaluate and verify NILM product performance, common test protocols and metrics are needed. But first, industry and researchers in the NILM field must converge on performance expectations for NILM. Once the expectations are clear, metrics and protocols can be developed that 1) are meaningful and of value to potential stakeholders, 2) empower researchers and developers to advance these technologies to desired levels, and 3) increase confidence in the product capabilities. As part of this project, Pacific Northwest National Laboratory intends to address these needs. The next steps are to engage industry stakeholders and an advisory board to reach agreement on common protocols and metrics that can be used to objectively evaluate performance and consistently compare appliance disaggregation technologies for different applications or uses. 


\section{Acknowledgments}

Pacific Northwest National Laboratory's work with NILMs has been funded by the U.S. Department of Energy Building America Program, The DOE Emerging Technology Program, the Bonneville Power Administration, and the State of Washington. We are grateful to these organizations for providing funding and guidance. The Northwest Energy Efficiency Alliance, and its contractor, Ecotope, provided access to the Residential Building Stock Assessment data and have helped to identify and resolve data issues. NILM manufacturers were identified to participate in the study. PNNL thanks them for their pioneering spirit and willingness to provide data and help in resolving operating and data issues throughout the study. 



\section{Acronyms and Abbreviations}

BPA

CT

DR

ELCAP

$\mathrm{kHz}$

MTU

NEEA

NIALM

NILM

PLC

PNNL

PT

RBSA

TED
Bonneville Power Administration

current transformer

Demand Response

End-Use Load and Consumer Assessment

kilohertz

measuring transmitting unit

Northwest Energy Efficiency Alliance

non-intrusive appliance load monitoring

non-intrusive load monitoring

power-line carrier

Pacific Northwest National Laboratory

potential transformer

Residential Building Stock Assessment

The Energy Detective 



\section{Contents}

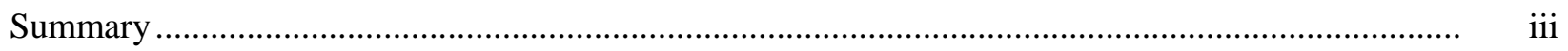

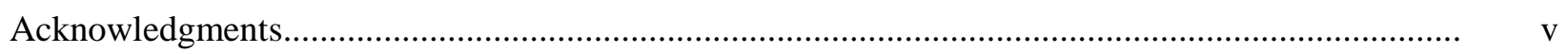

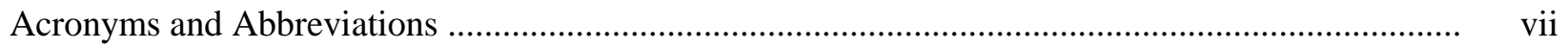

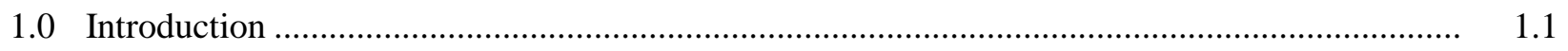

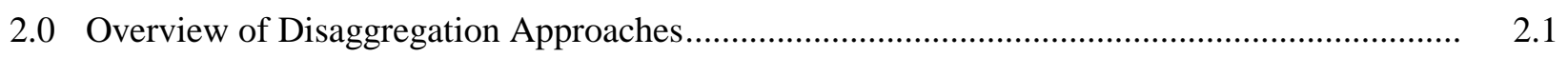

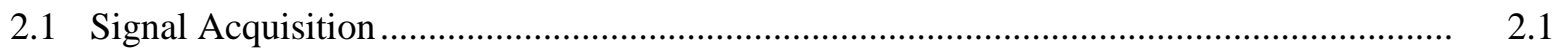

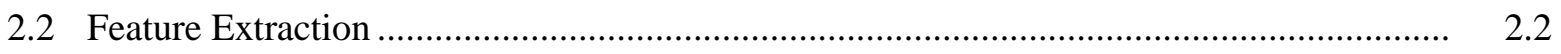

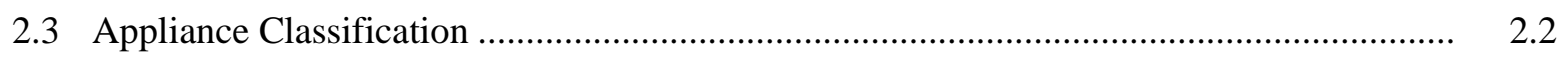

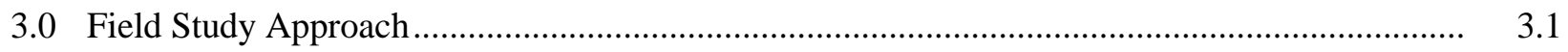

3.1 Background on NEEA RBSA Field Homes ................................................................ 3.1

3.2 Study Homes for NILM Evaluation ......................................................................... 3.2

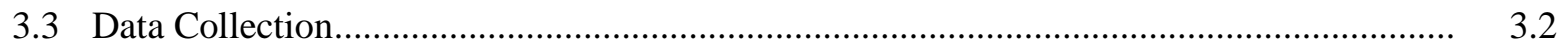

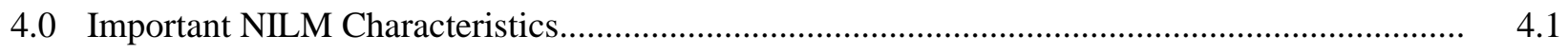

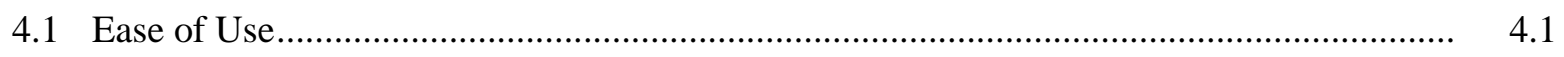

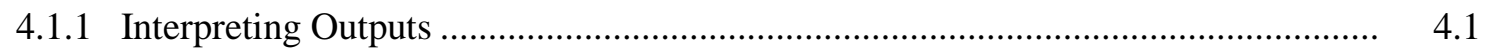

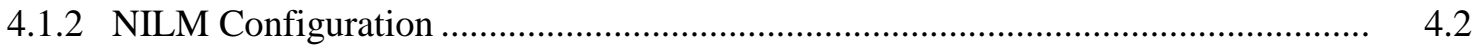

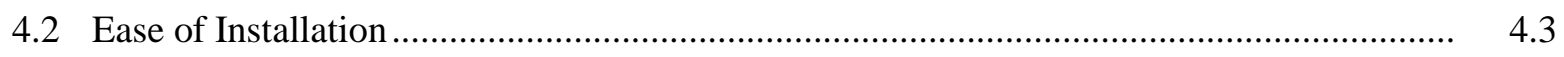

4.2.1 CT Sensors Installed in an Electrical Panel.......................................................... 4.3

4.2.2 Socket Sensors Installed on a Traditional Utility Meter ......................................... 4.4

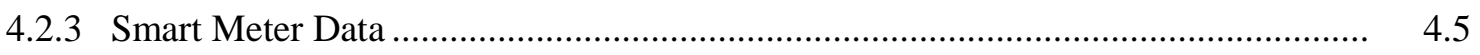

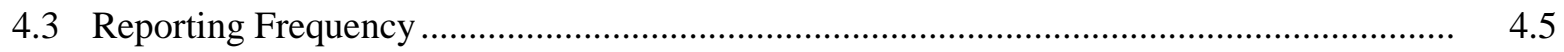

$5.0 \quad$ Performance Accuracy ............................................................................................ 5.1

5.1 Performance Accuracy Metrics …............................................................................ 5.1

5.2 Evaluation of Accuracy and Results .............................................................................. 5.3

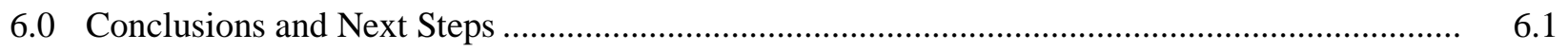

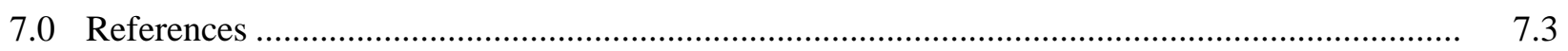

Appendix A - List of Active NILM Vendors ......................................................................... A.1 


\section{Figures}

2.1 Process of Appliance Disaggregation Using NILM ..........................................................

4.1 Typical Panel-Based Sensor Installation in Residential Electrical Panel (TED)...................4.4

5.1 Refrigerator Energy Profile (over 1 day) for Home 12063 .................................................5.2

5.2 NILM A Home 12063: NILM/Baseline Appliance Correlation Matrices.............................5.5

5.3 NILM A Home 13248: NILM/Baseline Appliance Correlation Matrices. .............................5.6

5.4 NILM Furnace/Baseline Refrigerator Energy Profile for Home 12063 ................................5.7

5.5 NILM Furnace Blower/Baseline Refrigerator Hourly Energy Profile for Home 12063 .........5.8

5.6 NILM Oven/Baseline Dryer Energy Profile for Home 14560 ..............................................5.9

5.7 NILM Dishwasher/Baseline Electric Resistance Heating Energy Profile for Home 13248..5.10 


\section{Tables}

5.1 NILM A: Accuracy of Energy Estimates for "As-Labeled" Appliance Pairs ........................... 5.4

5.2 NILM B: Accuracy of Energy Estimates for "As-Identified" Appliance Pairs ........................ 5.4

5.3 NILM A: Accuracy of Energy Estimates for "Relabeled" Appliance Pairs .............................. 5.7 



\subsection{Introduction}

With the widespread deployment of advanced metering infrastructure, 50 million smart meters were installed by July 2014, covering approximately $43 \%$ of the total households in the United States (Greentechgrid 2015a). With these smart meters, electricity service providers are able to obtain hourly and/or 15-minute whole-house-level energy-consumption data from customers. On average, each smart meter costs about $\$ 200$ and requires a specialized technician to install it (Hao et al. 2015). Moreover, the obtained metered data are mostly used for billing purposes, with few additional benefits offered to residential customers. However, the ability to disaggregate real-time appliance-level power consumption could have many additional benefits for consumers, electricity service providers, and society for energy conservation, energy efficiency, and demand response (DR) purposes if it were feasible and costeffective. Some example benefits include 1) increased awareness of energy use to empower consumers to make better decisions; 2) new options for automated commissioning, diagnosis, and fault detection of residential and small commercial buildings; 3) improved and simplified load studies leading to identification of specific end-use equipment, facilities, and locations that are candidates for DR and energy-efficiency programs; and 4) more efficient, cost-effective, and comprehensive quality assurance programs to verify savings from energy-efficiency measures and DR.

To obtain disaggregated appliance load data, the current practice is to sub-meter by installing a smart plug or power meter on each major appliance in the residence. However, the typical cost of a smart plug is about $\$ 50$ (Hao et al. 2015). In a typical home with 5-10 major appliances, the sub-metering cost is up to $\$ 500$, not including the additional cost of other hardware (e.g., gateway), software needed for providing services (e.g., data analytics), or labor necessary to install the meters. For this reason, the cost of traditional sub-metering is economically untenable for residential and commercial buildings. A more cost-effective monitoring approach is needed to capture the aforementioned benefits associated with such a widespread metering infrastructure. In addition to reduced cost, a viable metering device should be user-friendly, easy to install, and informative based on the specific application(s) for which it is being used. In this context, informative means whether disaggregated energy use reported to users (e.g., thirdparty applications, residential users, building operators, etc.) is frequent and accurate enough to empower reasonable decisions and actions to be taken.

Non-intrusive load monitoring (NILM) or non-intrusive appliance load monitoring (NIALM) is an analytic approach used to discern individual loads based on a single metering point as opposed to using traditional metering infrastructure at each appliance. NILM technologies have the potential to be costeffective, because of the reduced need for physical metering infrastructure. These technologies also have the potential to exhibit all of the aforementioned characteristics, which makes them a viable alternative to sub-metering. However, since the inception of the NILM concept in the 1980s, evaluations of these technologies have focused on reporting performance accuracy without investigating sources of inaccuracies or fully understanding and articulating the meaning of the metrics used to quantify performance. As a result, the market for, as well as, advances in these technologies have been slowly maturing. At this time, 18 active companies operating in the United States, United Kingdom, Ireland, and Canada have been identified to have commercially available or advanced prototypes of NILM (see Appendix A). Although a number of NILM products are now commercially available to homeowners and facility managers, their capabilities in terms of performance accuracy and suitability for specific applications are not yet understood. To stimulate the market for NILM as well as technological 
advancements, stakeholders and other interested parties should be kept aware of the evolving capabilities of NILM products and their readiness to be used for specific applications.

In 2013, Pacific Northwest National Laboratory (PNNL) and Northwest Energy Efficiency Alliance (NEEA) initiated a field study to evaluate the performance of existing NILM products in residential settings. The study was conducted by leveraging existing sub-metering infrastructure in the Residential Building Stock Assessment (RBSA) owner-occupied testbed that is located in the Pacific Northwest and operated by NEEA. At the time, the six candidate NILM products identified and acquired for the study were available for sale or at an advanced stage of development, capable of having their hardware safely installed within the field homes, and could return disaggregation results in a format needed for analysis. As a result of the field testing experience, PNNL and NEEA gained important insights into the performance accuracy of the studied NILM technologies and the NILM characteristics that appeal to stakeholders.

The purpose of this report is to present data and information about the characteristics and performance accuracy of commercially available NILM products included in the field study. But first, an overview of NILM disaggregation approaches is given in Section 2.0. Section 3.0 summarizes the field study conducted to evaluate the performance accuracy of the acquired NILM products. The characteristics of the NILM technologies evaluated in the assessment are then discussed in Sections 4.0, in terms of their ease of use, ease of installation, and reporting frequency. Performance accuracy results, based on the field study are presented in Section 5.0. Conclusions and next steps are presented in Section 6.0 . 


\subsection{Overview of Disaggregation Approaches}

Several comprehensive literature reviews of the research and development of NILM technologies have been presented in the literature (Armel et al. 2012; Zoha et al. 2012; Zeifman and Roth 2011; Butner et al. 2013; Kamilaris et al. 2014). In this section, only a brief overview of NILM approaches is given. As illustrated in Figure 2.1, the general process of NILM algorithms involves three steps: 1) acquisition of the whole-building load measurements; 2) appliance feature extraction, which involves pulling out features or patterns in the load measurement data that can be used to distinguish individual appliances; and 3) appliance classification, which requires analyzing the extracted features to discern appliancespecific load from the whole-building load data.

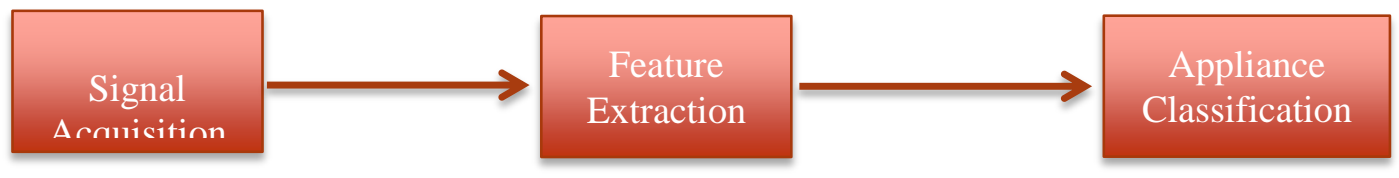

Figure 2.1. Process of Appliance Disaggregation Using NILM

\subsection{Signal Acquisition}

The first step, acquisition of whole-building-level power consumption data, is the prerequisite for any of the NILM algorithms. However, this information can be gathered using a variety of different variables (e.g., power, voltage, current, power factor, etc.), measurement device(s), measurement locations, and sampling frequencies. The most common measurements are the current and voltage at the entry point of a residence. Based on these two measurements, it is also easy to obtain the real power, reactive power, and power factor. Sometimes, advanced measurements such as harmonic distortion and electromagnetic interference are also required (Makonin 2012).

Regarding the different devices used, there are current-transformer-based devices that are installed in the breaker panels, utility-meter-based devices installed at the utility meter outside of a building, and smart meters that have been widely deployed in the United States. Inaccuracies in circuit-level measurements will have a strong influence on the disaggregation accuracy of NILM algorithms, so it is important for these measurements to have high levels of accuracy. Different independent system operators and utilities generally accept the use of meters that meet American National Standards Institute (ANSI) C-12 standards for revenue settlement and operational visibility purposes. According to ANSI C12 standards $^{1}$ (MacDonald et al. 2012), the absolute value of the metering error should be less than $2 \%$. Most current NILM products require the collected power consumption data to be uploaded to the vendors' servers, and disaggregation is performed at the server. The sampling rate of whole-house load data ranges from one sample per 15 minutes to $15 \mathrm{kHz}$ or even higher frequencies.

\footnotetext{
${ }^{1}$ Minimum Meter Accuracy table. http://images.masscec.com/uploads/programdocs/Production\%20Tracking\%20System/Minimum\%20Meter\%20Acc uracy\%20Requirements.pdf
} 


\subsection{Feature Extraction}

Step two, feature extraction, is a process of extracting features or signatures from the received signal. There are two main classes of appliance power signatures or features, steady-state and transient. Both approaches refer to identifying changes in the operation when an appliance modulates from one operational state or level to another, but the two approaches differ in what data they focus on. The steady-state appliance signature is one of the most widely used signatures in literature (Hart 1992; Wong et al. 2013). It refers to step changes in steady-state active and/or reactive power consumption levels. It is easy to use, does not require fast sampling data, and works well on large ON/OFF appliances such as water heaters and air-conditioners. The transient appliance signature refers to unique features such as the shape, duration, size, and harmonics of transient power fluctuations of appliances that can be used to distinguish different appliances. These short-term, transient fluctuations in power consumption occur immediately after changes in an appliance's operating state (e.g., from the OFF to ON state) and before a new steady-state is reached. Although the transient analysis gives more precise information, extracting this type of signature requires high-frequency data sampling. As a result, more resources are needed to construct and maintain a complex transient signature database. Besides the aforementioned two signature-based approaches, hybrid approaches have been proposed, which use a combination the steadystate and transient signatures for appliance disaggregation.

\subsection{Appliance Classification}

The final step, appliance classification, refers to analyzing features extracted from the whole-building load data to categorize specific appliances. In general, the NILM algorithms can be categorized as either event-based or non-event-based. Event-based NILM algorithms refer to approaches that rely on edge detection algorithms to detect occurrences of events, such as an appliance turning ON/OFF or a change in operating mode. The extracted features around the neighborhood of the event points are then classified using supervised machine learning algorithms such as support vector machines, artificial neural networks, Bayes classifier, and k-nearest neighbor clustering (Wong et al. 2013). Event-based algorithms typically require online or offline training processes to build a library for appliances from a priori labeled appliance power consumption data to train the appliance classifier. The training processes could need sub-metering or sophisticatedly designed experiments that might be labor-intensive and costly.

The unsupervised learning techniques, commonly used by non-event-based methods, have recently become popular in the NILM field and are more appealing and scalable than the supervised machine learning techniques from a practical point of view. However, unsupervised learning classifiers have not been implemented in a standard way in the literature (Makonin 2012). These algorithms do not rely on edge detection algorithms, but take every sample of the aggregate power signal received into account to infer individual loads (Wong et al. 2013). This causes the non-event-based method to be more computationally intensive than event-based methods. Although the algorithms are less computationally efficient, unsupervised machine learning methods (e.g., Hidden Markov Model) do not require prior knowledge of load behavior or patterns, so the process involving load libraries can be avoided. This makes them less labor-intensive and more cost-effective. Instead, the appliance loads are directly clustered and disaggregated based on whole-house power data collected. 


\subsection{Field Study Approach}

In 2013, PNNL and NEEA initiated a field study to characterize the performance of NILM technologies under "real-world" conditions. The study was conducted by leveraging existing submetering infrastructure in the Residential Building Stock Assessment (RBSA) owner-occupied testbed located in the Pacific Northwest and operated by NEEA. For the evaluation, PNNL and NEEA initially identified six candidate NILM products that were either already available for sale or at a sufficiently advanced stage of development, capable of having their hardware safely installed within the RBSA field homes, able to collect data over the internet, and able to return the disaggregation results for analysis. Four to six of each of the six identified NILM technologies were acquired and installed in a total of 30 homes.

As mentioned before, the NILM field is highly volatile as vendors continue to search for new ways to improve the technology and product configurations, expand market targets, and enable new applications. In addition, many of the companies are small startups with limited resources that require focus and direction. During the field study period, three of the six NILM companies found it necessary to focus on revamping and implementing major upgrades to their product or change company direction to expand their market base. Because many commercial products are set up to output summaries to the users through a dashboard, arrangements were initially made with the participating vendors to gain access to NILM disaggregation outputs. This meant some of the NILM vendors needed to invest time and resources in converting the data from their software package to a usable format. Therefore, when the three companies made a decision to change directions, they also found it necessary to withdraw from the field assessment, because they were unable to continue providing support.

Three vendors remained throughout the entire study and are denoted as Vendors A, B, and C herein. Their corresponding NILM products are denoted as NILM A, NILM B, and NILM C, respectively. Vendor A provided disaggregation results for four homes at 5-minute intervals over the course of the field study. Vendor B provided results for five homes, but declined to provide outputs at less than 24-hour intervals. Therefore, it was only possible to perform a partial analysis of its product. Vendor $\mathrm{C}$ participated using a developmental technology and did not have a process in place to quickly disaggregate and return the necessary results for the analysis. Consequently, a complete set of results could only be provided for one home and partial results for another. Also, data could only be provided at 24-hour intervals given the time constraints. Given the limited data obtained, the developmental product was not evaluated. The subsequent sections provide additional background on the NEEA RBSA field homes, the specific homes that were selected for participation in this NILM evaluation, and the characteristics of the data and data collection process.

\subsection{Background on NEEA RBSA Field Homes}

The End-Use Load and Consumer Assessment (ELCAP) project, which ran from 1983 to 1990, was a major end-use data collection project undertaken by Bonneville Power Administration (BPA) to support data needs for end-use load research, load management program planning and applications, load forecasting, and to identify resource conservation potential from new demand-side technologies or programs. ELCAP data sets, now more than 20 years old, are becoming increasingly dated, and consequently may no longer be as representative as they once were. To better understand new and emerging residential load profiles in the Pacific Northwest, the NEEA conducted the RBSA research 
study from 2011 to late 2014 to expand the existing ELCAP data set. In addition, RBSA collected data about the individual, appliance-specific load shapes, the occupant characteristics of residences, and fuel choices in the Pacific Northwest. The RBSA included billing analysis and field survey information for more than 1,400 homes that represent the diverse climates, building practices, and housing types across the Pacific Northwest region. As part of the RBSA, a representative sample of 100 homes, chosen throughout the region, were equipped with energy sub-metering equipment and equipment necessary to measure water flows, interior temperature and relative humidity, and lighting levels (Baylon et al. 2011). These homes had their energy use monitored on a 5-minute basis over a 2-year period between 2012 and 2014.

\subsection{Study Homes for NILM Evaluation}

Of the 100 monitored homes from the RBSA NEEA testbed, 30 homes were selected to receive NILM technologies as part of this NILM evaluation. NEEA chose the 30 homes because their demographics, building characteristics, and appliance inventories were representative of homes found in the Pacific Northwest region. Because the intention of this NILM study was to assess technology performance under "real-world" conditions with the homeowners behaving as they normally would, homeowners received no instructions, cues, or incentives to alter their behavior following the installation of a NILM technology. Detailed descriptions of each home, including occupant(s), appliance, and structure details, are available for each of the field homes in the report by (Baylon et al. 2011).

Each individual NILM technology was installed in approximately four to six individual homes between late 2013 and early 2014, and left in place to collect data until the RBSA homes were decommissioned in September 2014. Each home in the field study received only one NILM technology during installation to ensure that there were no interactions between technologies. Consequently, the official study period for some technologies began as early as late October 2013, while others were not installed until mid-January 2014. This resulted in some of the sites having data from an earlier date than others. In a few rare instances it was necessary to take both the NILM and the sub-metering equipment in individual field homes offline for one or more days. The dates of any outages were excluded from the study analysis, including days that may have only had a partial outage.

\subsection{Data Collection}

Within each home most electrical loads were equipped with metering equipment. The sub-metered data recorded average power consumption (in watts) for electrical loads present within each home over a 5-minute interval. These loads typically included heating, ventilation, and air-conditioning systems, hotwater heaters, appliances, televisions and television accessories, and computers and computer accessories, and may also have included other loads when present. Loads that were not included in the metering (but are included in the home's overall load) include small consumer electronics (i.e., hair dryers), infrequently used devices (i.e., portable electric space heaters), and other portable, miscellaneous loads. While lighting electrical data were collected during the RBSA, they were collected separately from the rest of the home's appliances and were not available at the time of this study. Home electrical use data collected by the sub-metering equipment were gathered remotely by NEEA's contractor, Ecotope, over a wireless $3 \mathrm{G}$ cellular network over the course of the study. These data for the 30 homes included in the NILM evaluation were provided to PNNL to use as baseline data. 
Data collected by the NILM devices were initially gathered by the respective hardware at each site and uploaded to the company's respective cloud-based server over the RBSA $3 \mathrm{G}$ network. Once the data from the homes were received, the server-based algorithms attempted to both identify what appliance loads were present in each home, as well as disaggregate the electricity consumed by each respective load (in kilowatt hours). The whole-house and appliance-level energy-consumption data were then provided to PNNL by each of the NILM vendors in comma-separated values (.csv) formatted files. Of the three NILM technologies in the study, disaggregation results for two of them were reported at 24-hour intervals (despite collecting the data at 1-minute intervals). Only one company reported results at 5-minute intervals. In instances where the NILM results were reported at 24-hour intervals, the 5-minute baseline data were aggregated up to the 24-hour period to facilitate comparison and analysis. 



\subsection{Important NILM Characteristics}

To accelerate the market for NILM technologies, there has to be confidence that the deployment will lead to benefits. This technology is still maturing and we need to be able to identify applications and environments where existing NILM technologies are useful now, as well as, areas of research and development that can help advance capabilities even further for future use. As mentioned previously, a number of characteristics are important to consider when evaluating the efficacy or appropriateness of a given NILM technology for a given application. These characteristics are ease of use, ease of installation, and reporting frequency. For example, a residential, consumer-based NILM should be easy to use and install, whereas a residential NILM that is operated by and intended primarily for a utility audience, can potentially have more complicated installation and use requirements.

\subsection{Ease of Use}

There are several ways an end-user may be required to interact with a NILM technology. End-users may include, but are not limited to, homeowners, commercial building owners, commercial building occupants, commercial building operators, energy managers, electricity service providers, and other thirdparty applications (i.e., building automation systems, diagnostic applications). These different types of end-users will have different capacities for interacting with the product and different capabilities for interpreting the disaggregated appliance-level energy use data in order to determine actions to be taken. In the following subsections, the necessary interactions with each technology involved in the NEEA field study and end-users are discussed, in addition to, possible pros and cons for different end-users.

\subsubsection{Interpreting Outputs}

All three technologies included in the NEEA field study were designed for residential users and feature web-based dashboards for NILM configuration and displaying disaggregation results. Through these dashboard interfaces, a user is presented with disaggregation information at a broad and generic level. Examples include daily power use of appliances being tracked, historical trends for individual appliances or the home's overall energy consumption, and the cost in dollars for appliance and home energy use over a day, week, or month. Such information is significantly more informative and actionable than the information that is typically presented to consumers on a monthly utility bill. By presenting more granular appliance-level data and historical information, users can be kept cognizant of the relative energy use of different appliances and actions having the biggest impact on their overall utility bill. As a result, homeowners can make more conscious decisions regarding the operation of their homes. In addition, homeowners can compare current appliance energy usage with historical information to understand how changes in behavior or appliance age may be affecting energy use and take corrective action. With this type of granular feedback alone, residential consumers have the potential to achieve energy savings of up to 12\% according to some studies (Ehrhardt-Martinez et al. 2010; Armel et al. 2013; Greentechgrid 2015b). It is also projected that energy savings of greater than $20 \%$ could be obtained if more insightful and actionable information were provided to the homeowners, such as alerting them to a particularly energy-intensive appliance, identifying appliances that may be faulty or failing, and identifying specific energy-saving steps. 
The NILM A product was designed with both the average homeowner and more sophisticated users in mind. The users of this product have the option to export disaggregated appliance data to standard file types (e.g., ASCII, Excel, etc.), as well as view graphical and summary reports via a user interface. The added capability to export the disaggregated results as time-stamped energy-use data files is of greater interest for commercial building operators or other parties responsible for managing multiple buildings. Exportable data, made available in such a flexible format, may allow for the data to be used by existing building automation control systems to better manage building efficiency. Also, the data may be easy to use and leveraged for more in-depth analysis by third-party energy managers and applications for purposes of reducing energy use. However, the raw disaggregated appliance data are beyond what typical single-family homeowners are willing or able to analyze and interpret. Because the software is loaded and run locally, the users themselves are also responsible for storing these data, which may also be beyond the capabilities of most individual homeowners, especially if records are kept over a prolonged period of time.

\subsubsection{NILM Configuration}

Appliance-level disaggregation requires a method to assist the algorithm in identifying loads and distinguishing individual appliances, which can be quite diverse from one building to another. Several approaches can be used to configure the algorithms for detecting and labeling loads. One example approach includes requesting the user to input limited information so that the loads in the building can be inferred by the NILM algorithm. Another approach is manual user training where a user is required to follow a sequence of steps to train the algorithm to identify each specific load and load signature. Another approach is to assume a certain level of user understanding of the loads in a building and rely on the users to identify specific loads by having them observe load profiles displayed through a dashboard. Depending on the configuration setup requirements of a user, the average homeowner may or may not have the time, interest, or necessary expertise to configure such a device to identify the loads correctly. In addition, some commercial building operators or managers of multiple buildings may find it costprohibitive to assign one or more workers to the task.

All three of the NILM technologies examined in the NEEA field study were generally designed to minimally engage with end-users, so they were set up to automatically recognize load features or patterns to disaggregate and label loads with little or no information requested from the user. This avoids requiring the users to conduct time-intensive training sessions and makes it more likely that the NILM will actually be installed and used. The NILM C device incorporates a web-based interface for presenting load disaggregation results to end-users with no configuration needs beyond those necessary to set up and connect the data gateway with the central cloud server. NILM B device also included web-based services, where the end-users are expected to respond to a brief survey to provide initial high-level details about their building and how it is typically used (e.g., number of people living in it, age of the building, etc.) to infer the loads that might exist on the premises. The NILM A technology offered a locally installed software program, which was designed for commercial and residential users. For configuration purposes, the user was requested to specify the building type (commercial, residential: single-family, or residential: multi-family) and to select appliances that exist in the facility from a list after being presented with the probabilities of certain appliances being present based on the building type selected. This information is likely requested to assist the NILM devices in labeling and classifying loads by precluding loads that are not present within the facility. For this product, the user has to confirm the list of appliances present, otherwise defaults are chosen. Selecting the wrong appliances or having a set of 
appliances or patterns not represented in the NILM load library could cause degradation in the performance of this NILM device.

At present, the NILM A and B devices examined in this report requested high-level information from users at the time of installation. While neither of these products included required manual user training during initial setup of the technology, this method of engaging the user for training disaggregation algorithms may also be most useful and acceptable as long as the instructions given are simple for targeted users to follow.

\subsection{Ease of Installation}

The whole-building load consumption data used for load disaggregation mainly come from one of three measurement sources: 1) current transformer (CT) sensors installed within the electrical breaker panel inside the home, 2) socket sensors installed on traditional utility meter outside the premise, or 3) cloud-based smart meter data. Each source would need to be connected to a gateway device (or datalogging device) for data collection and transmission. Depending on the measurement source the NILM technology relies on, the devices needed to obtain real/reactive power measurements may or may not be already installed at appropriate locations. This metering hardware may also be included with the NILM or may need to be purchased from a third-party vendor. For these reasons, the additional hardware needs and installation requirements for each case are two key factors that affect the cost of these NILM products, and in turn influence the consumers' willingness to purchase them. The NILM A device, included in the NEEA field study, relies on measurement source 1. The NILM B device is a softwareonly solution; options are offered to allow measurements to be obtained from source 1 or 3 . This allows whole-house power consumption data to be collected from either a panel-installed meter purchased from a third-party vendor or a pre-existing electric-utility smart meter. The NILM C device collects measurements based on source 2 . The following sections provide a detailed description of the hardware needs and installation requirements for each of the three types of NILM measurement sources.

\subsubsection{CT Sensors Installed in an Electrical Panel}

Most of the NILM vendors rely on electrical metering hardware that is installed in the electrical panel. This type of NILM product requires installation of properly sized CTs around the two service feeds to electrical panel, where the electrical mains connect to the breaker bus bars. In addition to the CTs, a voltage measurement device is needed to make phase-accurate power measurements. This voltage measurement device is sometimes referred to as a potential transformer (PT). A photograph of the CT sensors as installed in a home, are included as Figure 4.1. For this type of NILM device, a certified electrician is required to install the CTs at the power mains of the breaker panel and a measuring transmitting unit (MTU) or gateway that also contains the PTs needed. The MTU is used to transmit measurements collected to a communication hub in the building, through a building's electrical wiring, using power-line carrier (PLC) communication. This allows for measurement data to be collected and uploaded by the NILM platform. 


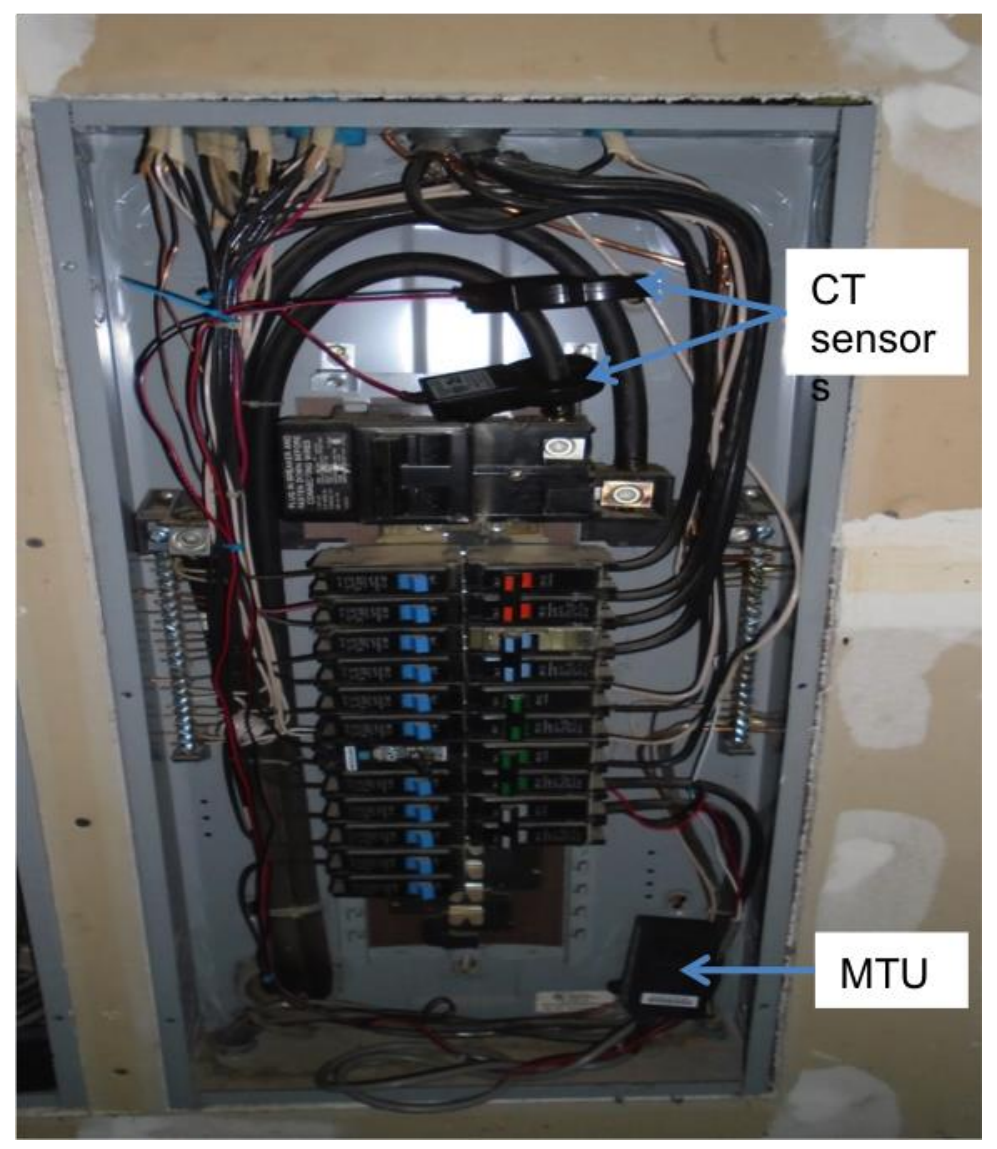

Figure 4.1. Typical Panel-Based Sensor Installation in Residential Electrical Panel (TED). CTs are installed at top on service feed; the MTU resides in the bottom right of panel.

\subsubsection{Socket Sensors Installed on a Traditional Utility Meter}

Some of the NILM vendors employ a meter-base or "socket" type sensor. The advantage of this type of NILM device is that metering is done outside the home on the "utility-side" of the meter and does not require access to the customer's electrical breaker panel. However, the installation of the socket sensor requires the serving electric utility to be engaged in the process. The socket sensor is designed to reside in between the utility meter and the meter base. This device contains all necessary components and electronics (CTs, PTs, and gateway) to measure and convey active/reactive power measurements. The installation process is initiated by the utility's metering staff to remove the existing meter and then confirm proper configuration of the socket sensor with that of the meter base. Of concern, is making certain the connection jaws in the meter base are compatible with the socket sensor. Once confirmed, the NILM socket meter is installed followed by the existing electrical utility meter. The utility representative then verifies successful installation by ensuring the existing meter is powered properly and is recording the expected readings.

Once the hardware is installed, the NILM devices that depend on CT sensors installed at the breaker panel or on socket sensors placed at the utility meter have similar configurations for data collection and access. In most cases, these NILM products have a means to connect to an existing wireless network 
within the home for data uploading and eventual customer access. Such a wireless network is required for data collection and transmission on these devices. Therefore, if the home does not have an existing wireless network that can be used for such a purpose, a dedicated wireless network may need to be set up. In some cases, this connection is through a direct wireless interface with the home's wireless router, while in other cases data are transferred from the socket sensor via the home's electrical wiring (a technology known as power-line carrier) to a gateway, from which the router is accessed. A properly connected sensor enables data to be uploaded at predetermined frequencies, from once a minute or hour, to once a day. As these data are uploaded, they are typically warehoused in remote data servers from which NILM software or cloud-based platforms can access the data to perform appliance disaggregation and report findings to end-users.

\subsubsection{Smart Meter Data}

With the advent of electric-utility smart meters, utility-derived interval data have become a source of whole-building power consumption data for NILM products. Typically, these smart meters are the property of the serving electric utility and thus are installed according to their specifications by their staff. The data collected by the utilities are generally hourly or 15-minute sampled energy-consumption data. Access to data is authorized based on an agreement between the utility, the utility account holder, and/or the NILM vendor. Data security, privacy, and access portals have all come under greater scrutiny in recent years because of increased cyber-attack activities. As such, this path to full customer data access for third-party NILM analysis can be a challenge. On the other hand, if smart meter data can be used no additional metering infrastructure is required, which makes such an approach attractive from a costeffectiveness and simplicity perspective.

Some NILM products employ another approach that relies on the utility smart meter, but utilitycollected meter data are not used. Instead, a gateway is used to enable frequent communication between smart meters, which are typically compatible with ZigBee communication protocols, and a cloud-based NILM platform. Currently, NILM platforms can also be permitted to access a consumer's utility smart meter through an agreement between the utility, the utility account holder, and/or the NILM vendor. Using such an intermediate device enables direct sampling of the meter readings at a faster rate than the utility-collected data (as fast as once every second). For this reason, this approach may be suitable for more applications requiring more granular measurement data.

Compared to the above two types of NILM products, the advantage of smart meter approaches is that there is no need to install extra sensors for load disaggregation. Therefore, it is much less labor-intensive, and does not require coordination with a certified electrician or utility metering electrician for installation if the smart meter is already installed on the home. Because of the minimal equipment and installation cost, the cost of these types of NILM devices will most likely be lower than the other products using CT or socket sensors.

\subsection{Reporting Frequency}

Another key characteristic that needs to be considered when deciding whether a disaggregation technology is appropriate for a particular application is the reporting frequency or the time interval at which data results are available from the NILM device. Current NILM vendors offer solutions with output rates of 1 second or slower. However, most are limited to 5-to 15-minute outputs. 
Because all applications do not demand the highly granular energy-use data, these technologies are not expected to report estimates at the highest possible granularity. Therefore, NILM technologies can be mapped to a particular range of applications depending on the limitations on output rate of energyconsumption estimates, in addition to performance accuracy and the other aforementioned important characteristics. For example, data analytics, for providing energy conservation advice to consumers, may only need 15-minute to hourly energy outputs of disaggregation algorithms. DR applications for delivering grid services at very short-term time scales (e.g., seconds) and long-term time scales (e.g., hours) may require 1-second and minute-by-minute energy information, respectively, for measurement and verification and/or automated control feedback needs. Providing outputs at multiple time frames could increase the practicality of a single NILM device being applied to a wide range of applications to inform decisions and monitor and verify performance. Or different NILM devices could be developed to meet these specialized needs at more appropriate price points for the given market segment. 


\subsection{Performance Accuracy}

Evaluating the performance of NILM technologies is not trivial because the performance could be misunderstood and misinterpreted if objective metrics and protocols are not used. Many of the loads being disaggregated by these technologies tend to be diverse and have unique characteristics. For example, multi-state loads such as refrigerator/freezers operate with compressors that cycle ON and OFF frequently to regulate compartment temperature. In addition, the defrost mechanism included draws more than two times the power of the compressor, but the defrost mechanism cycles less frequently (i.e., once every 6 to 24 hours depending on the design). There is a chance that a NILM device could label and represent the defrost mechanism and the compressor of a typical residential refrigerator/freezer in one of the following ways: 1) as a composite appliance, 2) as two separate appliances, 3) as a part of another appliance profile, or 4) not at all. In scenarios 2 and 3, it is possible that the NILM device has accurately represented the energy consumption of both appliances but just mislabeled them. In scenario 4, the NILM device is unable to isolate either function. This example illustrates that there are many factors that should be considered when describing the "accuracy" of NILM, and multiple protocols may be required to evaluate performance. To effectively identify the appropriate evaluation protocols, industry and other researchers in the field must first converge on performance expectations for NILM devices, because any defined protocols should evaluate the NILM device performance against an ideal or expected outcome. For example, should disaggregation performance be penalized if scenarios 2 and 3 occur? If so, then how? After the expectations are defined, a set of standardized metrics and protocols for characterizing NILM device performance can be developed to objectively compare the technologies. Recognizing the expectations will allow us to develop metrics and protocols that are meaningful and of value. In addition, researchers and NILM developers will have specific goals and objectives for advancing technologies in a unified way. In evaluating the NILM technologies included in the NEEA field study, the goal was to understand how well these products are able to track and estimate the energy use of individual appliances and identify sources of inaccuracy in performance. The specific metrics used in this evaluation and the results are presented in the ensuing sections.

\subsection{Performance Accuracy Metrics}

Because of the nascent nature of NILM technologies, at the time of this assessment there was not yet any consensus or broadly recognized methodology for measuring the accuracy of load disaggregation produced by these technologies. An initial literature review (Butner et al. 2013) found that a consistent set of NILM metrics for performance evaluation had not been previously documented in the existing literature. Additional interviews with product manufacturers also indicated that industry members had not yet converged upon a single set of methodologies for evaluating the performance of NILM technologies, nor had they disclosed any ongoing efforts to develop one. To conduct the analysis proposed under this study, it was necessary to develop a preliminary set of metrics that could be used to gauge the accuracy of load disaggregation. These metrics needed to be compatible with the nature and structure of the data outputs generated by the NILM products.

PNNL had previously developed a set of candidate metrics based on its literature review (Butner et al. 2013). However, when applying these metrics to the NILM and baseline (measured) energy-use data obtained from the field study, it was discovered that the metrics were unable to capture real-world 
performance attributes that were rather obvious visually. For example, the following candidate metric (from Butner et al 2013) was tested to determine NILM accuracy in estimating energy use:

$$
\text { energy accuracy }=1-\left(\frac{\text { measured energy }- \text { NILM energy }}{\text { measured energy }}\right)
$$

where measured energy is the energy measured by meter (watt-hours) over the defined study period and NILM energy is energy estimated by NILM (watt-hours) over the defined study period

For one home (12063), the energy accuracy of the NILM was computed to be $72.4 \%$ for a refrigerator, based on a 24 -week study period. Figure 5.1 shows the NILM and baseline energy-use profiles for the refrigerator in the home over a 24-hour period. From this figure, it is clear that the NILM device is not tracking the energy use of a refrigerator even though the energy accuracy was determined to be very high. In this case, the NILM device was actually reasonably tracking the energy-use profile of a hot-water heater. Because the water heater has large energy draws over a short duration that are comparable to the total energy consumption of a refrigerator over a longer term, the energy accuracy metric in Equation (5.1) could be misinterpreted if it were used to convey the NILM device performance. Therefore, the energy accuracy metric is an unsuitable metric for describing NILM device performance in accurately representing energy use of individual appliances.

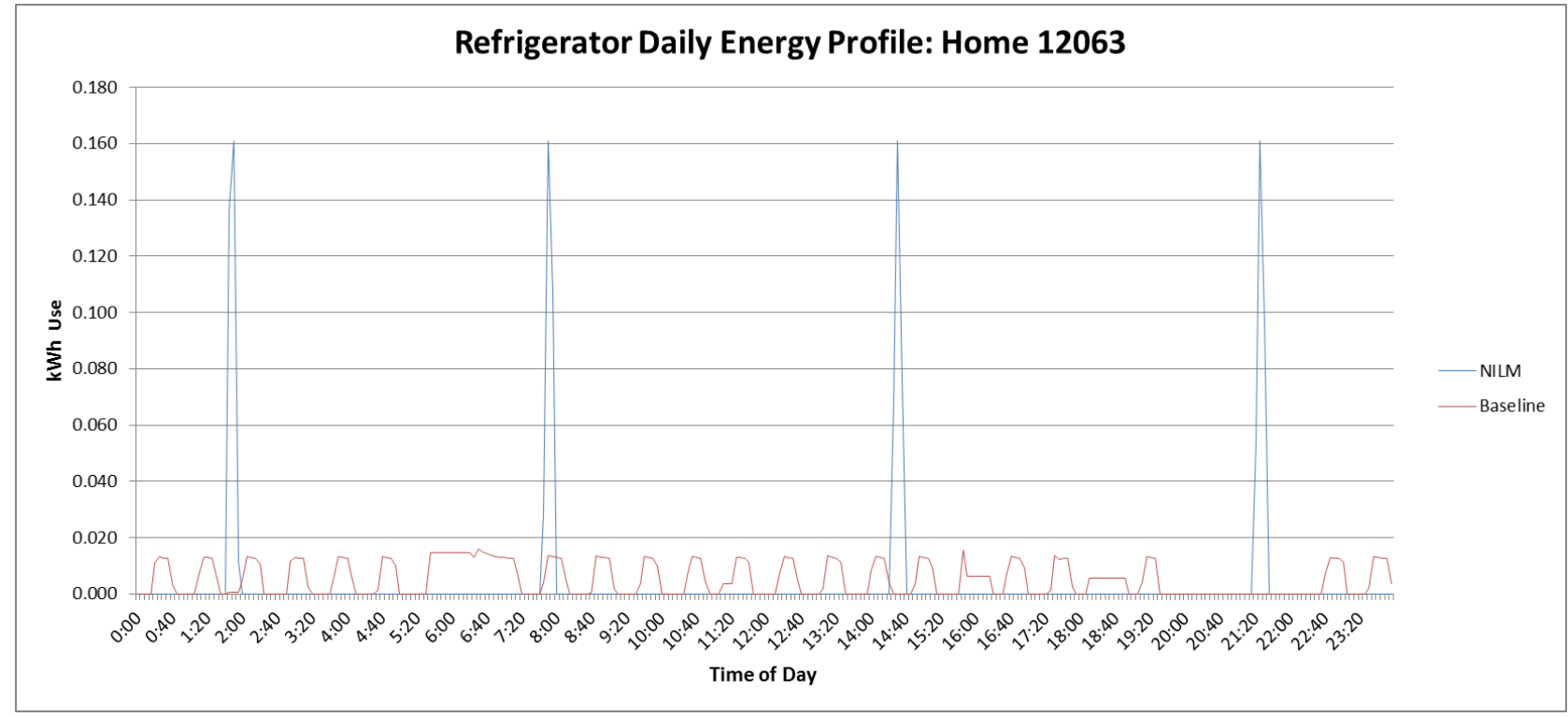

Figure 5.1. Refrigerator Energy Profile (over 1 day) for Home 12063.

After considering several metrics, the percent (\%) standard deviation explained by Nau (2015) was chosen to represent the accuracy of energy estimation,

$$
\% \text { standard deviation explained }=1-\sqrt{1-r^{2}}
$$

and 


$$
r^{2}=1-\frac{\sum\left(Y_{i}-Y_{i}\right)^{2}}{\sum\left(Y_{i}-\bar{Y}\right)^{2}}
$$

where $Y_{i}$ is the $i$ th observed value and $Y_{i}$ represents its corresponding predicted value. $\bar{Y}$ is the mean of the set of observed values. $r^{2}$ is the coefficient of determination, which indicates the fraction by which the variance of the errors between predicted and observed values is less than the variance of the observed values (Nau 2015). The closer $r^{2}$ is to 1 , the better the estimate is. Values less than zero indicate that the observed and predicted data are a poor match or not in agreement (Eisenhauer 2003). For the assessment of NILM device performance, the predicted values signify NILM device energy-use estimates and the observed values represent the measured energy use of the individual appliances identified by the RBSA sub-metering infrastructure. For the example given in Figure 5.1, $r^{2}$ was computed to be -13.6, which confirms the visual observation that the NILM device is not tracking the energy use of the refrigerator. Even though $r^{2}$ is able to indicate poor and good performance, it is more intuitive to interpret outputs in terms of the units the data are measured in (Nau 2015). For this reason, the percent standard deviation explained is used instead. This metric is able to capture accuracy in detecting events in the short-term (e.g., seconds and minutes), as well as, estimating energy use over a longer term (e.g., weeks). This intuition is further discussed in the following section, which discusses how this metric was applied to the NILM results obtained from the NEEA field homes.

\subsection{Evaluation of Accuracy and Results}

For each RBSA home in which each NILM A and B device was installed, NILM and baseline data sets for energy consumption of each appliance were accessed, checked for completeness, and binned into appliance-specific sets containing contiguous time-series values. Some data issues were found in both the NILM and baseline data sets. It was noted that a number of the data sets had missing values, data presented at different time intervals, and incorrect (e.g., "NA" or "\#Values") data entries. The problematic portions of these data sets were identified and removed. Once validated, contiguous data sets were assembled for each home by appliance with corresponding time stamps, the metric defined in Equation (5.2) for assessing the accuracy of energy estimates by the NILM was applied in a two-step process.

The purpose of the first step was to assess the accuracy of NILM device energy-use estimates for appliances that were labeled and reported by the NILM in each home. Therefore, the accuracy metric was applied to the NILM and the baseline "as-labeled" appliance pairs. These "as-labeled" appliance pairs are based on matching of the baseline appliance energy-consumption data obtained from the sub-metering with the corresponding NILM data obtained for that appliance. For instance, the "as-labeled" appliance pair for a refrigerator would consist of the NILM and the baseline energy-use data collected for the refrigerator in the home.

Table 5.1 and Table 5.2 present the accuracy of NILM energy estimates for "as-labeled" appliance pairs in each home in which NILM A and B products were installed. Only major appliances are considered in this analysis. The top row and first column of each table correspond to the major "aslabeled" appliance pairs considered and the list of homes the NILM product was installed in, respectively. "NA" entries (shaded in gray) indicate that the particular appliance was not monitored in the 
corresponding home. "ND" entries (shaded in yellow) denote cases where the particular appliance was monitored in the home, but was not detected by the NILM. As mentioned previously in Section 5.1, $r^{2}$ and hence the (\%) standard deviation explained metric used, can be negative if the observed (baseline data) and predicted (NILM data) are poorly correlated. The negative values computed for the appliance pairs in each home are reported as $0 \%$ in Table 5.1 and Table 5.2 and are in a red font. All other entries are the computed accuracy values for the appliance pairs in each home.

Table 5.1. NILM A: Accuracy of Energy Estimates for "As-Labeled" Appliance Pairs (4/19/14 to $10 / 29 / 14)$

\begin{tabular}{|c|c|c|c|c|c|c|c|c|c|}
\hline & $\begin{array}{c}\text { Clothes } \\
\text { Dryer }\end{array}$ & $\begin{array}{l}\text { Water } \\
\text { Heater }\end{array}$ & Refrigerator & Furnace & Dishwasher & $\begin{array}{l}\text { Clothes } \\
\text { Washer }\end{array}$ & Oven & Freezer & ER Heat \\
\hline $\begin{array}{l}\text { Home } \\
12063\end{array}$ & $0 \%$ & $85.1 \%$ & $0 \%$ & $0 \%$ & $0 \%$ & ND & NA & NA & ND \\
\hline $\begin{array}{l}\text { Home } \\
23384\end{array}$ & $12.5 \%$ & $0 \%$ & $0 \%$ & NA & ND & $0 \%$ & $5.6 \%$ & NA & ND \\
\hline $\begin{array}{l}\text { Home } \\
13248\end{array}$ & $77.1 \%$ & ND & $0 \%$ & NA & NA & ND & $0 \%$ & NA & ND \\
\hline $\begin{array}{l}\text { Home } \\
14560\end{array}$ & ND & ND & $24.9 \%$ & ND & $0 \%$ & ND & NA & $9.7 \%$ & NA \\
\hline
\end{tabular}

$\mathrm{ER}=$ electric resistance; ND = appliance was not detected by the NILM in the home; NA = appliance was not available in the home.

Table 5.2. NILM B: Accuracy of Energy Estimates for "As-Identified” Appliance Pairs (12/25/13 to $7 / 23 / 14)$

\begin{tabular}{|c|c|c|c|c|c|c|c|c|}
\hline & $\begin{array}{c}\text { Clothes } \\
\text { Dryer }\end{array}$ & $\begin{array}{c}\text { Water } \\
\text { Heater }\end{array}$ & Refrigerator & Dishwasher & $\begin{array}{l}\text { Clothes } \\
\text { Washer }\end{array}$ & Oven & Freezer & $\begin{array}{c}\text { ER } \\
\text { Heat }\end{array}$ \\
\hline $\begin{array}{l}\text { Home } \\
10040\end{array}$ & ND & $0 \%$ & $0 \%$ & NA & NA & ND & NA & $13.0 \%$ \\
\hline $\begin{array}{l}\text { Home } \\
12507\end{array}$ & NA & $0 \%$ & $0 \%$ & NA & NA & NA & NA & $25.7 \%$ \\
\hline $\begin{array}{l}\text { Home } \\
12994\end{array}$ & $0 \%$ & NA & $0 \%$ & NA & NA & NA & NA & NA \\
\hline $\begin{array}{l}\text { Home } \\
14284\end{array}$ & NA & $0 \%$ & $0 \%$ & NA & NA & NA & NA & NA \\
\hline $\begin{array}{l}\text { Home } \\
14577\end{array}$ & NA & $48.3 \%$ & $0 \%$ & NA & NA & NA & NA & NA \\
\hline
\end{tabular}

Evident in these tables, are several poor correlations in "as-labeled" appliance pairs, signifying that both NILM technologies are poorly tracking the energy use of most of the actual appliances they labeled. In addition, the NILM A device was unable to label or detect at least two major appliances in each home as indicated by the "ND" entries. There were not many major appliances monitored or available in the homes in which the NILM B device was installed. Across all of the homes and appliances available in the homes in which the NILM B device was installed, only two appliances were not detected by the NILM B device. However, the low-accuracy results obtained for the "as-labeled" appliance pairs for both NILM A devices, as shown in Table 5.1 and Table 5.2, primarily led the research team to review the data and search for missed correlations and errors in analysis. When visually inspecting the NILM energy outputs 
compared to the baseline sub-metered data, it was discovered that the NILM devices could possibly be reasonably estimating the energy use of another load that they mislabeled. However, to confirm this observation, a series of correlation matrices were generated for each home to help identify mislabeled loads by measuring the Pearson's R-square correlation coefficient (see Equation (5.3) in Section 0) between loads identified by the NILM technologies and the baseline data. Figure 5.2 and Figure 5.3 show the correlations between appliances labeled by the NILM A device and measured appliances in two homes, as examples. The appliances listed on the left vertical axes are the major appliances for which the NILM A device reported energy estimates. The appliances listed at the top on the horizontal axes are the appliances sub-metered in the corresponding home. Also, dark blue, white, and red indicate a strong correlation, no correlation, and very poor matching, respectively. In home 12063, there were two water heaters, DHW and DHW_2, as shown in Figure 5.2. It is apparent that the NILM device labeled one water heater correctly, because the Water.Heater/DHW matchup results in a R-square value close to 1 (indicated by the dark blue). However, the second water heater, DHW_2, was labeled as a refrigerator. Similarly, the clothes dryer is recognized as an oven, the furnace (Furn) as a clothes dryer, and the furnace blower as a refrigerator. As can be seen in Figure 5.3, there were no very strong correlations between labeled and sub-metered appliances in home 13248, as there were in home 12063. However, there is a chance that the electric resistance heating (ER_2) measured in home 13248 might be misclassified as a dishwasher by the NILM A device, based on the lighter blue indicator.

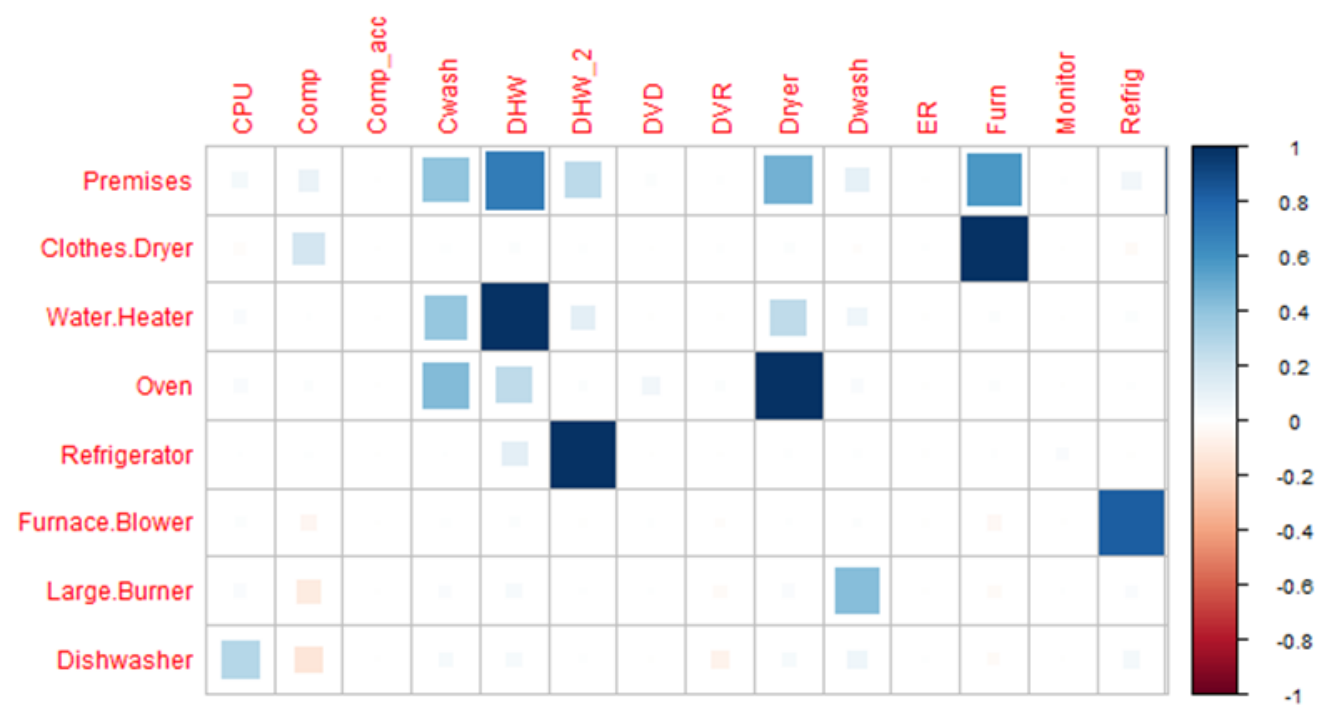

Figure 5.2. NILM A Home 12063: NILM/Baseline Appliance Correlation Matrices (4/19/14 to 10/29/14). Dark blue, white, and red indicate a strong correlation, no correlation, and a very poor match, respectively. 


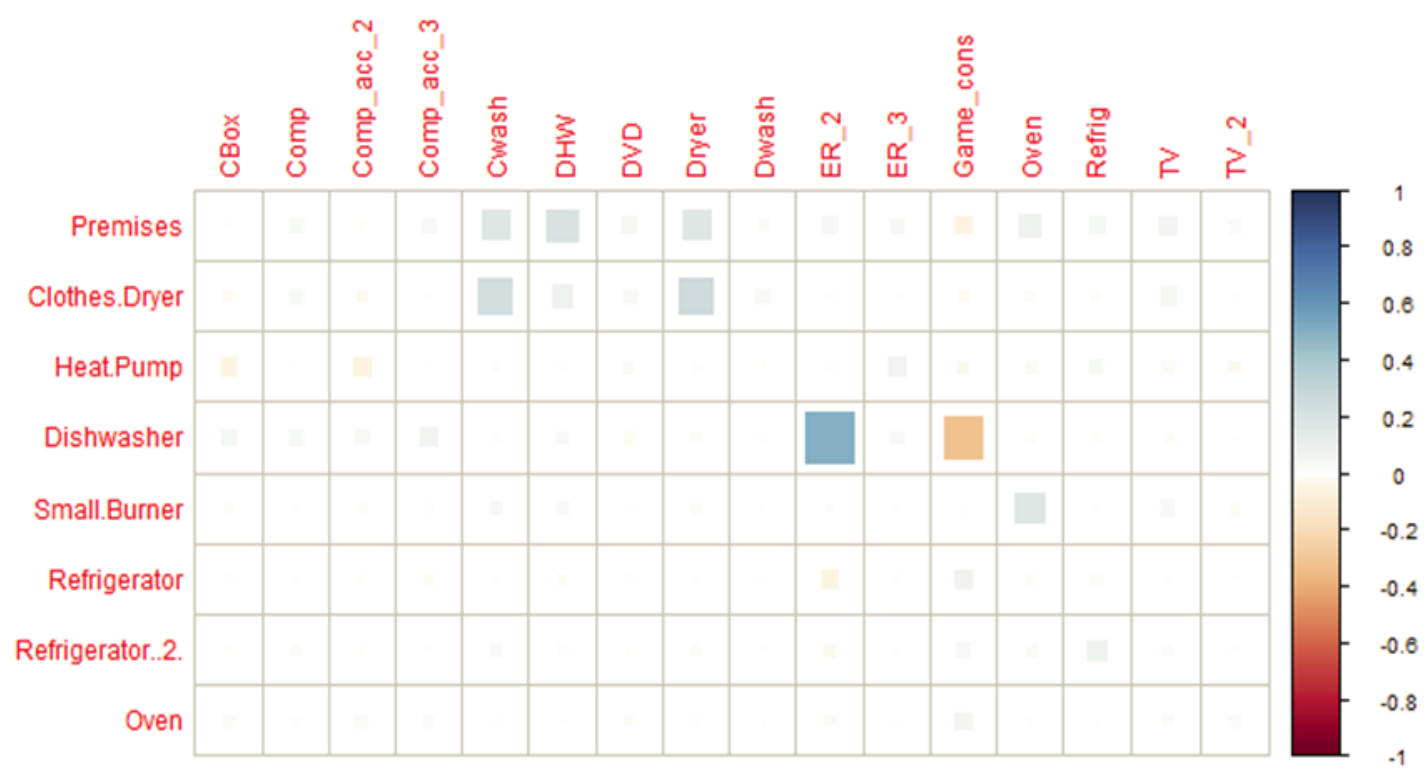

Figure 5.3. NILM A Home 13248: NILM/Baseline Appliance Correlation Matrices (4/19/14 to 10/29/14). Dark blue, white, and red indicate a strong correlation, no correlation, and very poor match, respectively.

After identifying these incorrect categorizations, the clearly identified loads mislabeled by the NILM technologies (as shown in Figure 5.2) were matched with the baseline appliance with which they were most correlated and relabeled as that baseline appliance. Then, the energy accuracy of each NILM device was reevaluated based on the same metric described in Equation (5.2) for all appliance matches found. This constitutes the second step of the evaluation. Table 5.3 presents the updated load disaggregation accuracy, based on the "relabeled" appliance pairs for the NILM A device. The additional values included (in bold), represent the accuracy computed after matching and relabeling the NILM data with correlated baseline appliance data. As shown, the accuracy of the NILM device in estimating the energy use of some appliances, significantly improved. Note that two water heaters instead of one were monitored and detected in home 12063 after relabeling the appliance pairs. Also, some of the appliances initially undetected by the NILM A device (see Table 5.1) were now being detected after re-matching some appliance pairs. The NILM A device initially mislabeled four of five appliances that were reasonably represented in one home. These results indicate an opportunity for better appliance identification strategies with certain appliances and applications. No additional correlated appliance pairs were found for the NILM B device. 
Table 5.3. NILM A: Accuracy of Energy Estimates for "Relabeled" Appliance Pairs (4/19/14 to $10 / 29 / 14)$

\begin{tabular}{|c|c|c|c|c|c|c|c|c|c|}
\hline & $\begin{array}{c}\text { Clothes } \\
\text { Dryer }\end{array}$ & $\begin{array}{l}\text { Water } \\
\text { Heater }\end{array}$ & Refrigerator & Furnace & Dishwasher & $\begin{array}{l}\text { Clothes } \\
\text { Washer }\end{array}$ & Oven & Freezer & $\begin{array}{c}\text { ER } \\
\text { Heat }\end{array}$ \\
\hline $\begin{array}{l}\text { Home } \\
12063\end{array}$ & $\mathbf{7 8 . 8 \%}$ & $\begin{array}{l}85.1 \% / \\
\mathbf{8 4 . 1 \%}\end{array}$ & $53.2 \%$ & $86.7 \%$ & $0 \%$ & ND & NA & NA & ND \\
\hline $\begin{array}{l}\text { Home } \\
23384\end{array}$ & $12.5 \%$ & $0 \%$ & $0 \%$ & NA & ND & $0 \%$ & $5.6 \%$ & NA & $81.4 \%$ \\
\hline $\begin{array}{l}\text { Home } \\
13248\end{array}$ & $77.1 \%$ & ND & $0 \%$ & NA & NA & ND & $0 \%$ & NA & $57.7 \%$ \\
\hline $\begin{array}{l}\text { Home } \\
14560\end{array}$ & $81.8 \%$ & ND & $24.9 \%$ & ND & $0 \%$ & ND & NA & $9.7 \%$ & NA \\
\hline
\end{tabular}

$\mathrm{ER}=$ electric resistance; ND = appliance was not detected by the NILM in the home; NA = appliance was not available in the home; bold font represents accuracy computed for matching and relabeling correlated appliance pairs.

In attempts to better understand correlations and accuracy, select data were reviewed graphically. Figure 5.4 presents the "relabeled" refrigerator appliance pair energy profiles over a 24-hour period. This correlation was found visually using correlation matrices generated (see Figure 5.4), where the NILM furnace data were found to align with the baseline refrigerator in home 12063. The NILM furnace data were then relabeled as a refrigerator. As the profile shows, once the proper correlation was made, the alignment and energy-use magnitude were very similar. One exception is the peak in energy use at about 5:45 AM. This peak, likely a refrigerator defrost event, was captured by the baseline metering but was either undetected by the NILM record or incorrectly categorized as or with another appliance. Another exception is the consistent underestimating of the energy use by the NILM device - on the order of roughly $10 \%$.

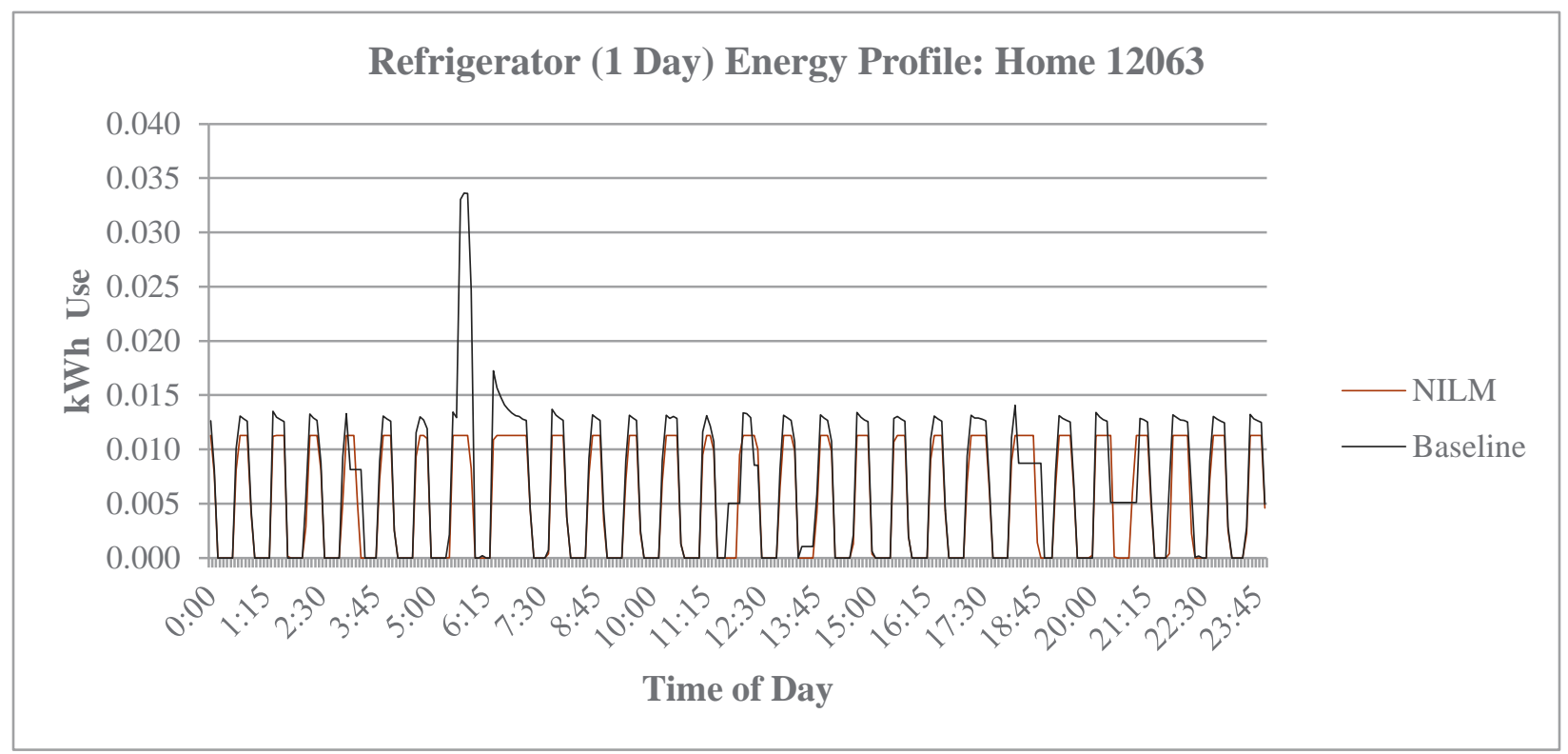

Figure 5.4. NILM Furnace/Baseline Refrigerator Energy Profile (over 1 day) for Home 12063

Both of these inaccuracies are better highlighted in Figure 5.5, which presents the first 7 hours of the same data set. As the figure illustrates, the alignment of refrigerator power draw events, including the magnitude and duration of the events, are quite good in most cases. Both Figure 5.4 and Figure 5.5 
highlight where these errors may be occurring and how they may be propagating through the data set due to consistent underestimation of the load and the missed defrost events that occur at least one time daily and consume relatively high levels of energy over 10- to 15- minute periods. Note also that the daily profile contains slight start and stop time offsets and, while they do not occur often, their presence is evident. After reclassifying the misidentified furnace blower as a refrigerator, the calculated energy accuracy was $51.2 \%$ for the 24-week period of study between 4/19/2014 and 10/4/2014. This result appears to be reasonable due to consistent underestimation of the load, missed defrost events that occur at least one time daily and consume relatively high levels of energy over 10- to 15-minute periods, and offsets in runtimes between the NILM device estimates and the measured energy use. For the case of the refrigerator, both of these issues offer opportunities for future study about classifying loads and improving energy estimates.

Another visual example is shown, in Figure 5.6, which depicts a "relabeled" dryer appliance pair pairing over 1 day. For this case, the NILM device energy data for an oven were found to be highly correlated with the baseline dryer in home 14560. The profile shows that all events were detected, but the NILM device again underreports, especially peak energy consumptions (at 9:00 AM). For this situation, the energy accuracy was calculated to be $\sim 82 \%$ over the 24 -week period of study, which makes sense because the error between the NILM and baseline data for the dryer varies and propagates throughout the period of study.

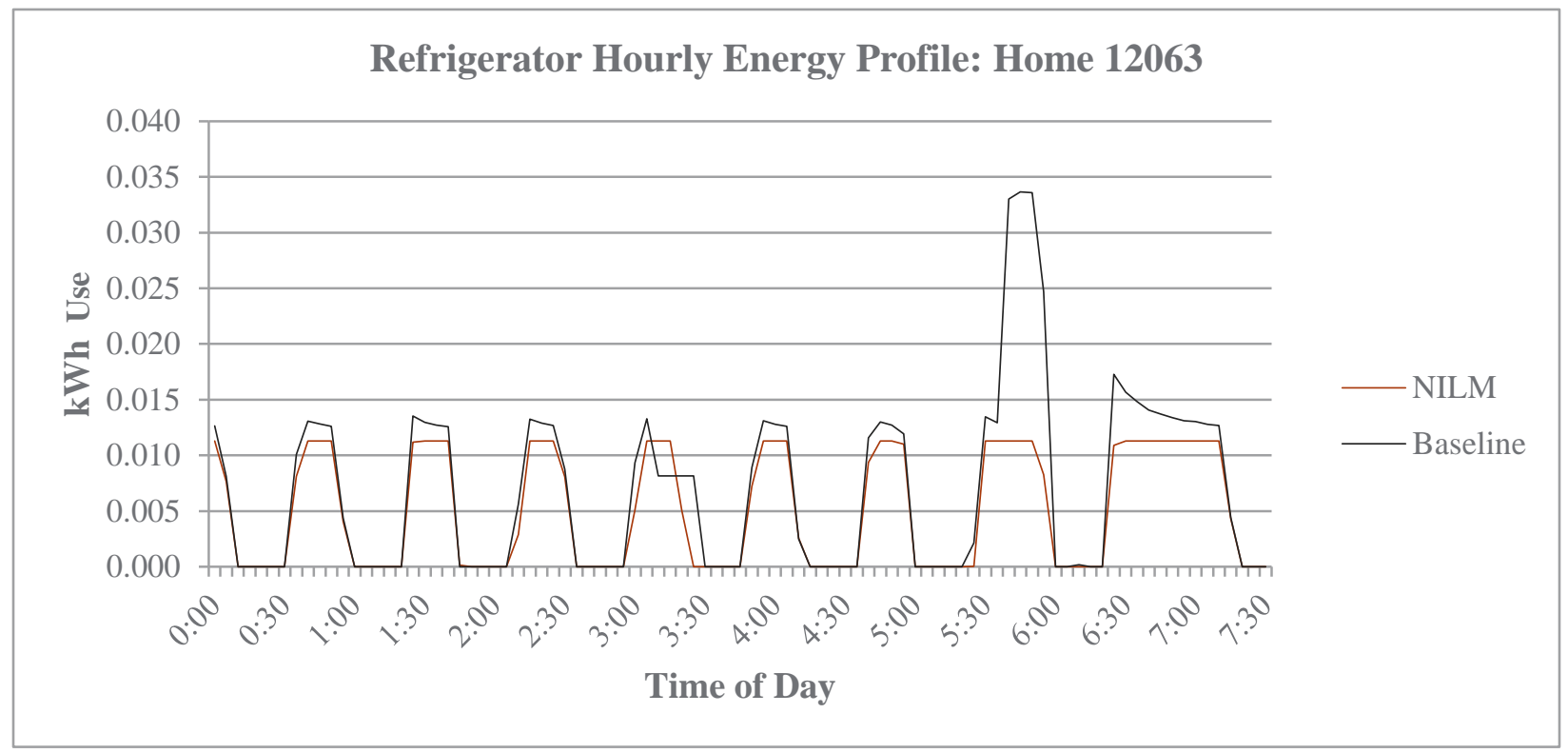

Figure 5.5. NILM Furnace Blower/Baseline Refrigerator Hourly Energy Profile (over 7.5 hours) for Home 12063 


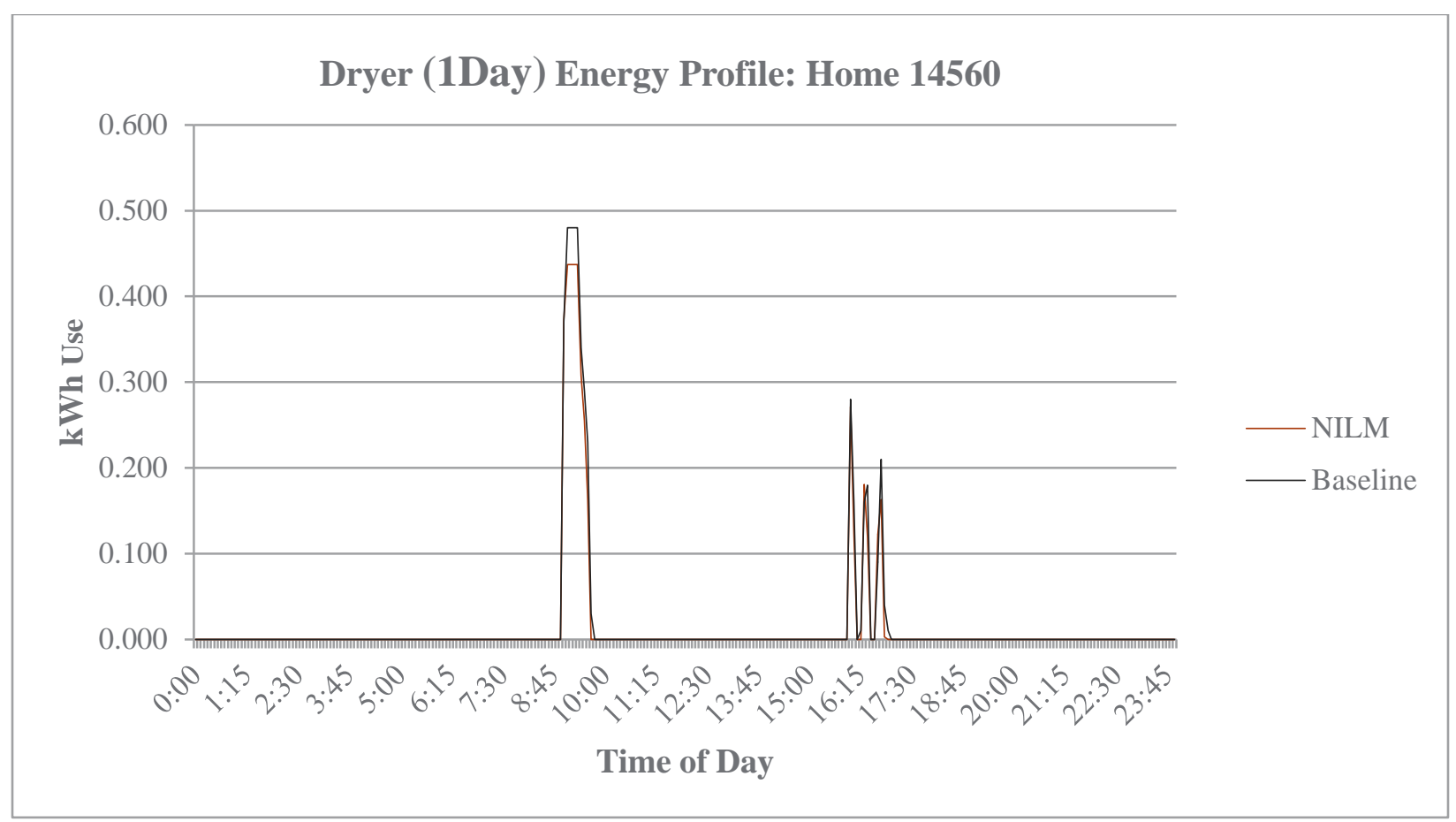

Figure 5.6. NILM Oven/Baseline Dryer Energy Profile (over 1 day) for Home 14560

In summary, the NILM A device was reasonably accurate at estimating energy consumption and event identification of resistive loads (i.e., electric resistance water heaters, heating, clothes dryers, etc.), but initially struggled with load identification. This NILM technology mislabeled at least one appliance in each home in which it was installed. In addition, it was unable to detect or track the energy use of at least three of the major appliances considered in every home in which it was installed. However, once the correct appliances were identified via re-matching and relabeling correlated appliance pairs, NILM device energy-consumption accuracy improved significantly. NILM A was more successful with ON/OFF type loads, such as the electric resistance water heaters, clothes dryer, and furnace; it had an overall energy estimate accuracy of $\sim 70 \%$ across all homes (after correction and excluding the uncorrelated appliance pairs where the NILM labeled appliances could not be re-matched with other correlated baseline appliances).

For multi-state loads, such as refrigerators and freezers, the average accuracy of the NILM A device was only $\sim 30 \%$ (after correction), indicating that some improvement is possible in better classifying appliances with multiple energy-consumption states. In this assessment, the NILM device was penalized for not recognizing the defrost cycles and consistently underestimating compressor cycles and offsets in ON/OFF runtimes of the compressor. The NILM A device also appeared to sometimes identify multiple loads as one appliance or report erroneous loads, which drastically decreased accuracy in those cases. For example, Figure 5.6 shows the energy-use profiles for a "relabeled" appliance pair between the NILM device's originally labeled dishwasher and an electric resistance heating load in home 13248. It is clear that the NILM device is tracking energy use of the actual load at some periods and estimating significant load at times when electric resistance heating is observed to be on. In this case, energy accuracy was observed to be $\sim 58 \%$, based on the 24 -week period of study. Based on the two stages of analysis performed so far, it is unclear whether the reason for degradation in performance is due to an actual or erroneous load being categorized with the freezer by the NILM device. Depending on the performance 
expectations for using NILM technologies for different applications, the former reason may be acceptable. If so, the NILM device performance should not be penalized. Another stage of analysis may be needed to understand these types of caveats and to report accuracy based on new appliance matches. This example shows how performance accuracy can be misinterpreted when additional analysis is not performed to examine reasons for the performance levels reported. Also, it illustrates the need for industry and stakeholders to converge on clearly defined performance expectations for NILM technologies to determine whether additional analysis is needed to assess accuracy based on misclassification of appliance loads.

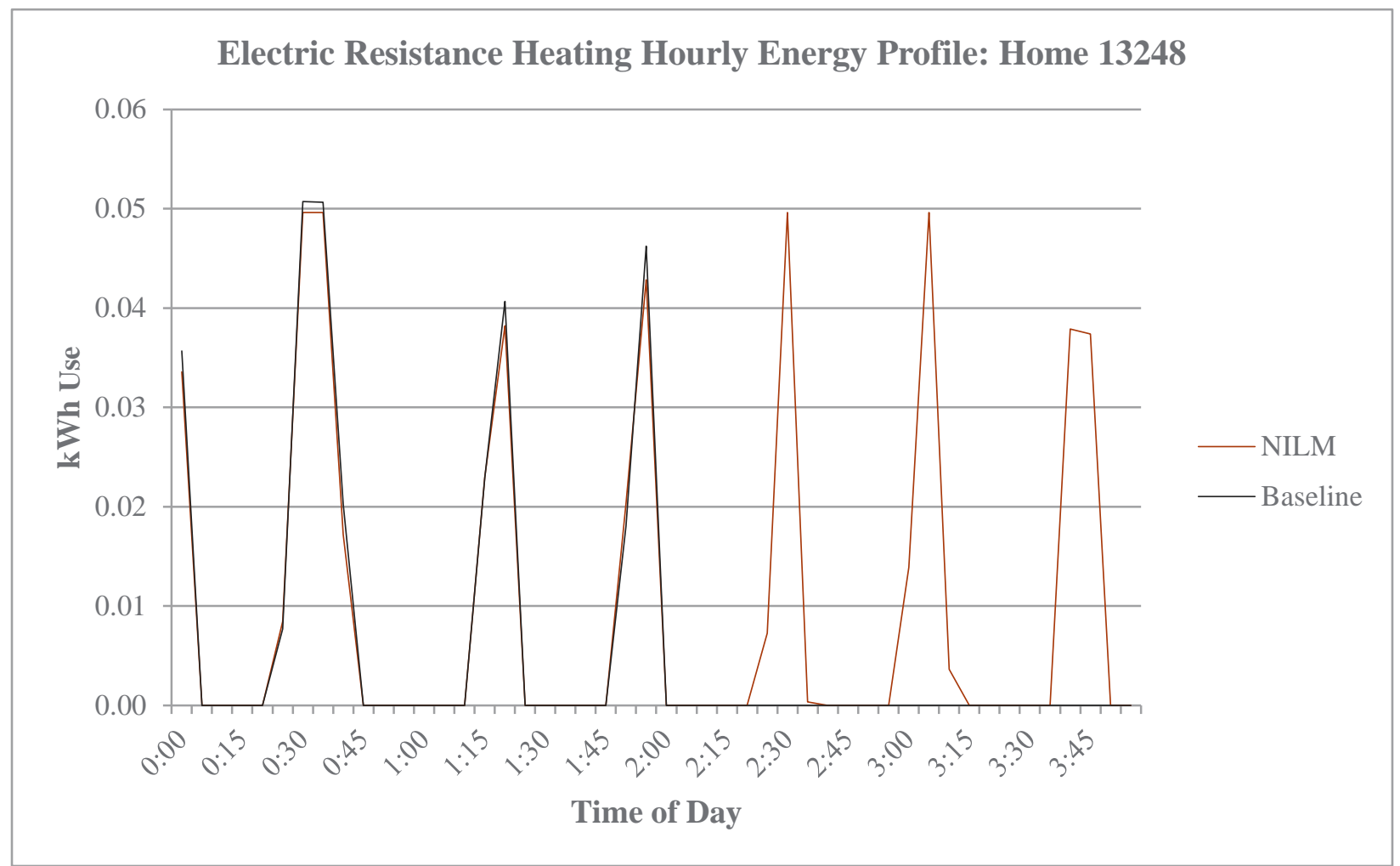

Figure 5.7. NILM Dishwasher/Baseline Electric Resistance Heating Energy Profile (over 4 hours) for Home 13248

Vendor B was only willing to provide 24-hour interval energy estimates for the performance evaluation. The NILM B device reported energy estimates for up to three appliances in either home, mainly recognizing them as a water heater, refrigerator, air conditioner, or dryer. For each home in which the technology was used, no additional correlated appliance pairs were identified. The energy accuracy was found to be less than $45 \%$ for all appliances identified. Due to the low resolution of the data received, the sources of inaccuracy cannot be further explored.

The analysis and results from this field study provide insight into the challenges of characterizing the performance of NILM technologies, the sources of inaccuracy, as well as development opportunities that should be explored to mature these technologies to be used to enable useful applications. 


\subsection{Conclusions and Next Steps}

Every NILM end-user and application does not require the same level of accuracy, power/energy-use output resolution, ease of use, and ease of installation (which impacts costs). Likely, in the future, a variety of unique NILM technologies will be developed and customized with a suite of characteristics that are acceptable for particular applications.

From the NEEA field test evaluation of a few existing NILM technologies, several insights were gained regarding the ease of use, installation requirements, and reporting frequency of available NILM technologies. The ease of use will likely affect the market adoption of such technologies, particularly in the residential consumer market, while the ease of installation has important considerations for the overall cost of the product. The reporting frequency influences the ability of the NILM data to be useful for particular applications.

While different levels of accuracy may be acceptable depending on the application, performance accuracy will continue to be a key consideration when considering and comparing different NILM technologies. However, evaluating the performance of NILM technologies is not trivial because the performance is easily misunderstood and misinterpreted. Previous performance studies have discouraged market uptake and decreased confidence in the potential value of NILM technologies because meaningful and objective metrics and protocols were not used to convey the suitability of the technologies for certain applications. In addition, the reasons for inaccuracy are not typically reported, so they cannot be used to guide and focus innovation in this area to enable a wide range of applications.

To evaluate technology performance, PNNL evaluated existing metrics that were previously proposed in the literature. PNNL found that selection of new metrics was warranted because candidate metrics were inflexible and unable to capture NILM device performance in a robust and meaningful way. After selecting a more comprehensive metric, the results were analyzed and some sources of performance inaccuracies in estimating energy use for these technologies were explored. For instance, for one NILM product, it was evident that the appliance classification process, used to identify individual loads, could be improved to significantly increase the accuracy of energy estimates of individual loads. Also, in some cases there was consistent underestimation of appliance energy use, which indicates the need to enhance the methods used to calculate energy use for certain appliances. More investigation is needed to identify other significant sources of inaccuracies and potential opportunities for improvement.

This report describes the preliminary evaluation of several commercially available NILM technologies. To encourage innovation in NILM technologies, researchers and stakeholders need to continue to be kept aware of their evolving performance capabilities and key characteristics that make them suitable for certain applications and market segments. Roughly 18 currently active NILM companies have advanced prototypes or commercially available products across the United States, United Kingdom, Ireland, and Canada. The market for these NILM technologies is slowly maturing. Further development of a robust set of performance metrics that is vetted with industry, communication about performance capabilities based on these metrics, and demonstration of other important characteristics are meaningful activities to pursue. They will help further the development of this new and emerging field by identifying applications where existing NILM technologies may be of benefit now, and by emphasizing areas of research that can help to advance NILM capabilities to a level that is appropriate for other applications. To accomplish these goals, industry and researchers in the field must first converge on a set 
of performance expectations for NILM devices. Clearly articulated and unambiguous expectations will then allow for development of metrics and protocols that 1) are meaningful and of value to the industry, 2) empower researchers and developers to advance these technologies to desired levels, and 3) increase confidence in the product capabilities. As part of an ongoing project, primarily sponsored by BPA ${ }^{1}$, PNNL plans to engage stakeholders and an advisory board to reach agreement on common protocols and metrics that are intended to be voluntary and used to objectively evaluate and compare appliance disaggregation technologies for different applications and uses.

\footnotetext{
${ }^{1}$ BPA TIP 327: NILM Accuracy Test Standard Development and Measurement Improvement
} 


\subsection{References}

Armel KC, G Dupta, G Shrimali, et al. 2013. "Is disaggregation the holy grail of energy efficiency? The case of electricity." Energy Policy 52:213-234.

Baylon D, P Storm, K Geraghty, et al. 2011. Residential Building Stock Assessment: Single-Family Characteristics and Energy Use. URL: http://neea.org/docs/reports/residential-building-stockassessment-single-family-characteristics-and-energy-use.pdf?sfvrsn $=8$

Berges ME, E Goldman, HS Matthews, et al. 2010. "Enhancing Electricity Audits in Residential Buildings with Nonintrusive Load Monitoring." Journal of Industrial Ecology 14(5):844-858.

Butner RS, DJ Reid, M Hoffman, et al. 2013. Non-Intrusive Load Monitoring Assessment: Literature Review and Laboratory Protocol. PNNL-22635, Pacific Northwest National Laboratory, Richland, Washington.

Ehrhardt-Martinez K, KA Donnelly, and JA Laitner. 2010. "Advanced metering initiatives and residential feedback programs: a meta-review for household electricity-saving opportunities." American Council for an Energy-Efficient Economy, Washington, D.C.

Eisenhauer JG. 2003. "Regression Through the Origin." Teaching Statistics 25(3):76-80.

Greentechgrid. 2015a (accessed). "50 Million US Smart Meters and Counting.” URL: http://www.greentechmedia.com/articles/read/50-million-u.s.-smart-meters-and-counting

Greentechgrid. 2015b (accessed). “Startup Smappee Goes Deep With Its Energy Disaggregation.” URL: http://www.greentechmedia.com/articles/read/startup-smappee-goes-deep-with-its-energy-

disaggregation?utm_source=feedburner\&utm_medium=feed\&utm_campaign=Feed\%3A+GreentechMedi $\underline{\mathrm{a}+(\text { Greentech+Media) }}$

Hart GW. 1992. "Nonintrusive appliance load monitoring." Proceedings of the IEEE 80.12:1870-1891.

Hao H, BM Sanandaji, K Poolla, et al. 2015. "Potentials and Economics of Residential Thermal Loads Providing Regulation Reserve." Energy Policy 79:115-126.

Kamilaris A, B Kalluri, S Kondepudi, et al. 2014. "A literature survey on measuring energy usage for miscellaneous electric loads in offices and commercial buildings." Renewable and Sustainable Energy Reviews 34:536-550.

MacDonald J, P Cappers, D Callaway, et al. 2012. "Demand Response Providing Ancillary Services A Comparison of Opportunities and Challenges in the US Wholesale Markets." Grid-Interop Forum 2012.

Makonin S. 2012. "Approaches to Non-Intrusive Load Monitoring (NILM) in the Home." PhD dissertation, Simon Fraser University, Burnaby, British Columbia.

Nau RF. 2015 (accessed). "Percent of standard deviation explained." URL:

http://people.duke.edu/ rnau/rsquared.htm 
Wong YF, YA Sekercioglu, T Drummond, et al. 2013. "Recent approaches to non-intrusive load monitoring techniques in residential settings." IEEE Symposium on Computational Intelligence Applications In Smart Grid (CIASG).

Zeifman M and K Roth. 2011. "Nonintrusive appliance load monitoring: Review and outlook." IEEE Transactions on Consumer Electronics 57 (1):76-84.

Zoha A, A Gluhak, MA Imran, et al. 2012. "Non-intrusive load monitoring approaches for disaggregated energy sensing: A survey.” Sensors 12 (12):16838-16866. 


\section{Appendix A}

\section{List of Active NILM Vendors}

Several non-intrusive load monitoring (NILM) products are commercially available for residential and/or commercial building segments. Table A.1 lists some active NILM vendors and their products, as well as the applications, current market targets, and the costs quoted from the vendors (if available). Table A.2 lists active NILM vendors and the specific measurement devices, measurement sampling rates and reporting frequency limits for their NILM products. Yellow highlights represent prototypes and ongoing research institutions where the products have not yet been made commercially available.

Table A.1. List of Active NILM Vendors and Their Products

\begin{tabular}{|c|c|c|c|c|}
\hline Vendor & $\begin{array}{l}\text { Product } \\
\text { Name }\end{array}$ & Applications & $\begin{array}{c}\text { Current Market Targets } \\
\text { and Users }\end{array}$ & Cost \\
\hline AlertMe & AlertMe & $\begin{array}{l}\text { Energy analytics, } \\
\text { appliance control, and } \\
\text { home automation }\end{array}$ & $\begin{array}{l}\text { Utilities, service } \\
\text { providers, retailers, } \\
\text { appliance } \\
\text { manufacturers, and } \\
\text { residential customers }\end{array}$ & $\mathrm{NA}$ \\
\hline Belkin & $\begin{array}{l}\text { NA } \\
\text { prototype }\end{array}$ & $\begin{array}{l}\text { Appliance } \\
\text { disaggregation }\end{array}$ & NA & Target at $\$ 200$ range \\
\hline Bidgley & $\begin{array}{l}\text { HomeBeat } \\
\text { Energy } \\
\text { Monitor }\end{array}$ & $\begin{array}{l}\text { Appliance itemization, } \\
\text { solar disaggregation, } \\
\text { event notification and } \\
\text { reminders, targeted } \\
\text { marketing, social } \\
\text { sharing, measurement } \\
\text { and verification, } \\
\text { energy-efficiency } \\
\text { programs, and } \\
\text { customer services }\end{array}$ & $\begin{array}{l}\text { Utilities, and residential } \\
\text { customers }\end{array}$ & $\begin{array}{l}\text { TED 5000: } \$ 200, \\
\text { Rainforest } \\
\$ 100, \quad \text { EAGLE: } \\
\text { ConnectPort: } \$ 120\end{array}$ \\
\hline $\begin{array}{l}\text { Blue Line } \\
\text { Innovations }\end{array}$ & $\begin{array}{l}\text { PowerCost } \\
\text { Monitor }\end{array}$ & $\begin{array}{l}\text { Appliance } \\
\text { disaggregation }\end{array}$ & $\begin{array}{l}\text { Homeowners, small } \\
\text { business owners, } \\
\text { utilities }\end{array}$ & $\$ 115$ \\
\hline $\begin{array}{l}\text { Energy Aware } \\
\text { Technology } \\
\text { Inc. }\end{array}$ & Neurio & $\begin{array}{l}\text { Appliance } \\
\text { disaggregation }\end{array}$ & Residential customers & $\$ 250$ \\
\hline Enetics & SPEED & $\begin{array}{l}\text { Appliance } \\
\text { disaggregation }\end{array}$ & Residential customers & $\$ 1,300$ \\
\hline EEme & EEme & $\begin{array}{l}\text { virtual home energy } \\
\text { audit analytics for } \\
\text { demand side }\end{array}$ & $\begin{array}{l}\text { Utilities and residential } \\
\text { consumers }\end{array}$ & NA \\
\hline
\end{tabular}


management

\begin{tabular}{|c|c|c|c|c|}
\hline $\begin{array}{l}\text { Fraunhofer } \\
\text { CSE }\end{array}$ & NA & $\begin{array}{l}\text { Load disaggregation } \\
\text { for energy } \\
\text { management }\end{array}$ & $\begin{array}{l}\text { Residential and } \\
\text { commercial customers }\end{array}$ & NA \\
\hline Load IQ & Enable.EI & $\begin{array}{l}\text { Energy disaggregation } \\
\text { and energy efficiency }\end{array}$ & $\begin{array}{l}\text { Commercial buildings } \\
\text { as well as industrial } \\
\text { facilities }\end{array}$ & $\begin{array}{l}\text { It is in pre-commercial } \\
\text { stage, one year rental } \\
\text { price of } \$ 2,000\end{array}$ \\
\hline $\begin{array}{l}\text { Navetas } \\
\text { Energy } \\
\text { Management }\end{array}$ & Loop & $\begin{array}{l}\text { Energy monitoring and } \\
\text { smart data analytics }\end{array}$ & $\begin{array}{l}\text { Residential customers. } \\
\text { However, the } \\
\text { disaggregation } \\
\text { technique has not been } \\
\text { implemented in the } \\
\text { current Loop system }\end{array}$ & $\begin{array}{l}£ 35 \text { for Loop energy } \\
\text { savings kit, Service fee: } \\
\text { first year free, then } £ 5 \\
\text { annually }\end{array}$ \\
\hline ONZO & ONZO & $\begin{array}{l}\text { Customer value } \\
\text { discovery from smart } \\
\text { meter data: energy } \\
\text { efficiency, customer } \\
\text { engagement, improved } \\
\text { targeting of sales and } \\
\text { marketing }\end{array}$ & Residential customers & NA \\
\hline Plotwatt & Plotwatt & $\begin{array}{l}\text { Data analysis, activity } \\
\text { alerts, and } \\
\text { recommendations on } \\
\text { rate plan selection, and } \\
\text { peak energy use }\end{array}$ & $\begin{array}{l}\text { Residential customers } \\
\text { and restaurants }\end{array}$ & $\begin{array}{l}\text { Free for residential } \\
\text { customers, } \$ 99 \text { capital } \\
\text { cost plus } \$ 99 \text { monthly } \\
\text { service charge for } \\
\text { restaurants }\end{array}$ \\
\hline Powersavvy & Powersavvy & Energy saving & $\begin{array}{l}\text { Residential customers } \\
\text { and business owners }\end{array}$ & $\begin{array}{l}\text { Monitoring a typical } \\
\text { home for a week, giving } \\
\text { an analyzed report costs } \\
€ 50\end{array}$ \\
\hline Qualisteo & $\begin{array}{l}\text { Lynx } \\
\text { Wattseeker }\end{array}$ & $\begin{array}{l}\text { Appliance } \\
\text { disaggregation }\end{array}$ & $\begin{array}{l}\text { Supermarkets, train } \\
\text { station, industrial site, } \\
\text { restaurants, offices, } \\
\text { shopping center, etc. }\end{array}$ & NA \\
\hline Smappee & Smappee & $\begin{array}{l}\text { Appliance } \\
\text { disaggregation }\end{array}$ & Residential customers & $\$ 250$ \\
\hline Verdigris & Verdigris & $\begin{array}{l}\text { Appliance } \\
\text { disaggregation }\end{array}$ & Residential customers & NA \\
\hline Verlitics & $\begin{array}{l}\text { Energy } \\
\text { Insight } \\
\text { Cloud } \\
\text { Service }\end{array}$ & $\begin{array}{l}\text { Appliance } \\
\text { disaggregation }\end{array}$ & $\begin{array}{l}\text { Home owners, business } \\
\text { owners, facility } \\
\text { managers, utilities, and } \\
\text { device manufacturers }\end{array}$ & NA \\
\hline
\end{tabular}




Wattics Wattics

Table A.2. List of Active NILM Vendors and Their Technology Types

\begin{tabular}{|c|c|c|c|}
\hline Vendor/Product & Measurement Devices & $\begin{array}{l}\text { Measurement } \\
\text { Sampling Rate }\end{array}$ & Output Rate \\
\hline AlertMe & $\begin{array}{l}\text { Energy Analytics is only a } \\
\text { software service solution. } \\
\text { It takes smart meter data } \\
\text { from utilities. }\end{array}$ & NA & NA \\
\hline Belkin & Bespoke sensor & $>1 \mathrm{MHz}$ & $<1$ second \\
\hline $\begin{array}{l}\text { Bidgley/ HomeBeat } \\
\text { Energy Monitor }\end{array}$ & $\begin{array}{l}\text { Smart meter and traditional } \\
\text { utility meter }\end{array}$ & NA & 5 minutes \\
\hline $\begin{array}{l}\text { Blue Line Innovations/ } \\
\text { PowerCost Monitor }\end{array}$ & $\begin{array}{l}\text { Utility-meter-based optical } \\
\text { sensor }\end{array}$ & NA & NA \\
\hline $\begin{array}{l}\text { Energy Aware } \\
\text { Technology Inc./ Neurio }\end{array}$ & Current transformer & NA & Not specified \\
\hline Enetics/SPEED & $\begin{array}{l}\text { Utility-meter-based } \\
\text { devices }\end{array}$ & 32 samples /cycle & $5 \mathrm{~min}$ \\
\hline EEme/EEme & Smart meter & 15 minute & NA \\
\hline Fraunhofer CSE & $\begin{array}{l}\text { Sensor installed in the } \\
\text { main breaker level of a } \\
\text { residence or commercial } \\
\text { establishment }\end{array}$ & $\begin{array}{l}1 \mathrm{~Hz} \text { (low } \\
\text { frequency } \\
\text { approach) or 50- } \\
100 \mathrm{kHz} \text { (high- } \\
\text { frequency } \\
\text { approach) }\end{array}$ & NA \\
\hline Load IQ/ Enable.EI & $\begin{array}{l}\text { Electrical panel-level } \\
\text { sensor }\end{array}$ & $3 \mathrm{k} \mathrm{Hz}$ & 1 second \\
\hline $\begin{array}{l}\text { Navetas Energy } \\
\text { Management/ Loop }\end{array}$ & $\begin{array}{l}\text { The sensor comes with the } \\
\text { kit, which attaches to the } \\
\text { main electricity feed. }\end{array}$ & NA & Not specified \\
\hline ONZO/ ONZO & $\begin{array}{l}\text { Data analysis providers } \\
\text { that takes smart meter or } \\
\text { utility customer data }\end{array}$ & NA & NA \\
\hline Plottwatt/ Plottwatt & Software-solution only & NA & 5 minutes \\
\hline Powersavvy/Powersavvy & $\begin{array}{l}\text { Powersavvy's own meter. } \\
\text { It is connected to the } \\
\text { electricity supply. }\end{array}$ & NA & NA \\
\hline $\begin{array}{l}\text { Qualisteo/ Lynx } \\
\text { Wattseeker }\end{array}$ & $\begin{array}{l}\text { Split-core current sensors } \\
\text { installed in the electrical } \\
\text { panel boards }\end{array}$ & $>1 \mathrm{kHz}$ & $<10$ minutes \\
\hline
\end{tabular}




$\begin{array}{llll}\text { Smappee/ Smappee } & \text { Current transformer } & 1 \mathrm{kHz} & 1 \text { minute } \\ \text { Verdigris/ Verdigris } & \text { Current transformer } & \text { NA } & \text { NA } \\ \text { Verlitics/EICS } & \text { Bespoke sensor } & \text { NA } & \text { NA } \\ \text { Wattics } & \begin{array}{l}\text { A 12-channel meter } \\ \text { installed on large } \\ \text { distribution boards }\end{array} & \text { NA } & \text { NA }\end{array}$




\section{Distribution}

No. of

Copies

\# Name

Organization

Address

City, State and ZIP Code

\# Organization

Address

City, State and ZIP Code

Name

Name

Name

Name

Name (\#)

\# Name

Organization

Address

City, State and ZIP Code
No. of

\section{Copies}

\# Foreign Distribution

\# Name

Organization

Address

Address line 2

COUNTRY

\# Local Distribution

Pacific Northwest National Laboratory

Name

Name

Mailstop

Mailstop

Name

Mailstop

Name

Mailstop

Name

(PDF) 




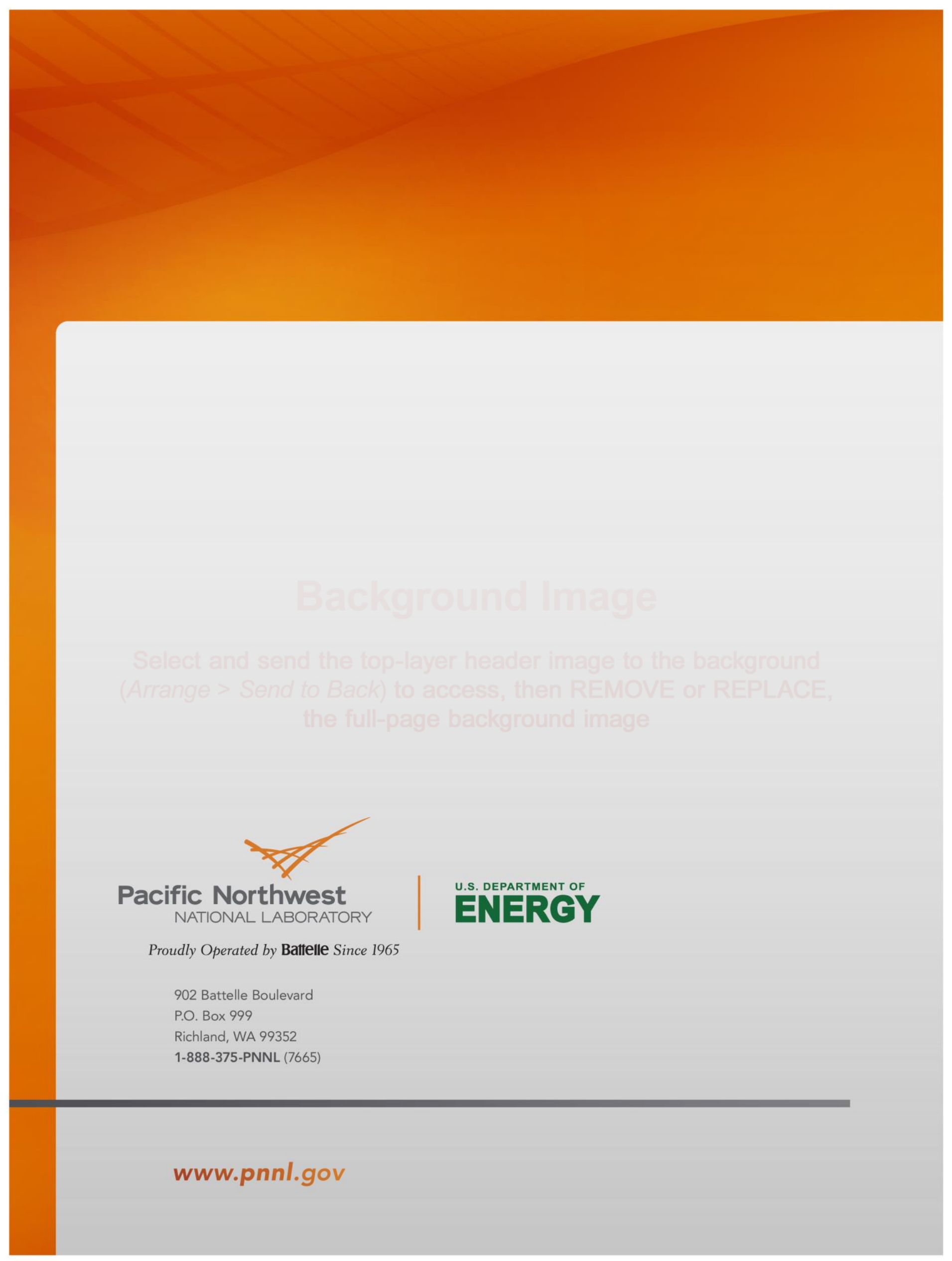

